\title{
Optimal bilinear control of eddy current equations with grad-div regularization
}

Yousept, Irwin

This text is provided by DuEPublico, the central repository of the University Duisburg-Essen.

This version of the e-publication may differ from a potential published print or online version.

DOI: https://doi.org/10.1515/jnma-2015-0007

URN: urn:nbn:de:hbz:464-20180829-120743-1

Link: https://duepublico.uni-duisburg-essen.de:443/servlets/DocumentServlet?id=46804

Legal notice:

(C) 2015 by Walter de Gruyter Berlin/Boston.

Source: J. Numer. Math. 2015; 23 (1):81-98. Published online: 07.08.2015. DOI: 10.1515/jnma-2015-0007

The publishers version is available at https://doi.org/10.1515/jnma-2015-0007

www.degruyter.com 
Irwin Yousept*

\section{Optimal bilinear control of eddy current equations with grad-div regularization}

Abstract: An optimal bilinear control problem governed by time-harmonic eddy current equations is considered to estimate the electric conductivity of a 3D bounded isotropic domain. The model problem is mainly complicated by the possible presence of non-conducting materials in the domain. We introduce an optimal control approach based on grad-div regularization and divergence penalization. The estimation for the electric conductivity obtained by solving the optimal control problem is allowed to be discontinuous. Here, no higher regularity property can be derived from the corresponding optimality conditions. We analyze the approach and present various numerical results exhibiting its numerical performance.

Keywords: PDE-constrained optimization, identification problem, eddy current equations, discontinuous electric conductivity, grad-div regularization, divergence penalization.

MSC 2010: 35Q61, 78A25, 78A30

DOI: 10.1515/jnma-2015-0007

Received August 6, 2012; accepted September 13, 2013

\section{Introduction}

Eddy current equations consist of a coupled system of first-order partial differential equations arising from Maxwell's equations by neglecting the displacement current. In the low frequency domain, they provide a reasonable approximation for the full Maxwell equations. We refer to the recent monograph by Alonso and Valli [1] for the analysis and modeling of eddy current equations. Let us consider the following time-harmonic formulation:

$$
\left\{\begin{aligned}
\operatorname{curl} \mu^{-1} \operatorname{curl} \mathbf{y}+\mathrm{i} \omega \sigma \mathbf{y}=\mathbf{j} & & \text { in } \Omega \\
\operatorname{div} \mathbf{y}=0 & & \text { in } \Omega \\
\mathbf{y} \times \mathbf{n}=0 & & \text { on } \Gamma
\end{aligned}\right.
$$

where $\Omega$ is a bounded Lipschitz domain in $\mathbb{R}^{3}$ with a connected boundary $\Gamma$. The vector field $\mathbf{y}$ denotes the magnetic vector potential, $\mathbf{j}$ the applied current source, $\mu$ the magnetic permeability, $\sigma$ the electric conductivity, $i$ the imaginary unit, $\omega>0$ the frequency, and $\mathbf{n}$ is the unit outward normal to the boundary $\Gamma$. In our context, $\Omega$ consists of isotropic heterogeneous materials, and the magnetic permeability $\mu$ is assumed to be known data satisfying

$$
0<\mu_{\min } \leqslant \mu(x) \leqslant \mu_{\max }<\infty \text { a.e. in } \Omega .
$$

Our goal is to estimate the electric conductivity of $\Omega$, which is typically nonsmooth and admits jump discontinuities. Engineering applications for such a parameter estimation problem can be found in optimal design of electromagnetic materials or in the context of electromagnetic metamaterials, related also to the control of electromagnetic waves (see [6, 21]).

Given a desired field (target) $\mathbf{y}_{d}$, the electric conductivity can be estimated by solving the following optimal control problem:

$$
\left\{\begin{array}{l}
\min \quad \frac{1}{2} \int_{\Omega}\left|\mathbf{y}-\mathbf{y}_{d}\right|^{2} \mathrm{~d} x+\frac{\varkappa}{2} \int_{\Omega} \sigma^{2} \mathrm{~d} x \\
\text { s.t. (1.1) and } 0 \leqslant \sigma(x) \leqslant \sigma_{\max } \text { a.e. in } \Omega
\end{array}\right.
$$


with a Tikhonov regularization parameter $\varkappa>0$, and an upper bound $\sigma_{\max }>0$. Note that the minimization problem (1.3) is nonconvex and features a bilinear structure. Here, the three-dimensional complex-valued vector function $\mathbf{y}$ is the state, whereas the scalar real-valued function $\sigma$ is the control. If a pair $\left(\mathbf{y}^{\star}, \sigma^{\star}\right)$ solves (1.3), then the optimal control $\sigma^{\star}$ provides an approximation for the electric conductivity.

The problem (1.3) is mainly complicated by the possible presence of nonconducting materials in the domain $\Omega$. Consequently, we have to allow controls that can take a zero value at any point in $\Omega$. This is the reason for including the Coulomb gauge

$$
\operatorname{div} \mathbf{y}=0 \quad \text { in } \Omega
$$

in the state equation (1.1). Notice that neglecting (1.4) would lead to non-uniqueness of the state, as every admissible control $\sigma$ can take a zero value. The presence of the Coulomb gauge, however, complicates the numerical realization of (1.1). Here, a nontrivial finite element discretization with divergence-free elements is required. We introduce therefore another formulation of the optimal control problem, where (1.4) is excluded from the state equation, but considered as an explicit equality constraint of the optimization problem. Furthermore, a grad-div regularization term $\hat{c} \nabla \operatorname{div} \mathbf{y}$, with a fixed constant $\hat{c}>0$, is included in the state equation. The corresponding optimal control problem reads as

$$
\min J(\mathbf{y}, \sigma):=\frac{1}{2} \int_{\Omega}\left|\mathbf{y}-\mathbf{y}_{d}\right|^{2} \mathrm{~d} x+\frac{\varkappa}{2} \int_{\Omega} \sigma^{2} \mathrm{~d} x
$$

subject to

$$
\left\{\begin{aligned}
\operatorname{curl} \mu^{-1} \operatorname{curl} \mathbf{y}-\hat{c} \nabla \operatorname{div} \mathbf{y}+\mathrm{i} \omega \sigma \mathbf{y}=\mathbf{j} & & \text { in } \Omega \\
\mathbf{y} \times \mathbf{n}=0 & & \text { on } \Gamma
\end{aligned}\right.
$$

and

$$
\begin{array}{r}
\operatorname{div} \mathbf{y}=0 \quad \text { in } \Omega \\
0 \leqslant \sigma(x) \leqslant \sigma_{\max } \text { a.e. in } \Omega .
\end{array}
$$

By the explicit equality constraint (1.6), every feasible state $\mathbf{y}$ of (P) satisfies $\hat{c} \nabla \operatorname{div} \mathbf{y}=0$, and hence it solves the original curl-curl problem:

$$
\operatorname{curl} \mu^{-1} \operatorname{curl} \mathbf{y}+\mathbf{i} \omega \sigma \mathbf{y}=\mathbf{j} \text { in } \Omega .
$$

Let us remark that the state equation (1.5) involves a curl-curl and grad-div structure. This is a well-known regularized formulation for Maxwell's equations (cf. $[1,19])$. We note that the choice of the positive constant $\hat{c}$ is arbitrary for our analysis.

The nonlinear optimal control problem (P) admits at least one optimal solution, provided that the corresponding feasible set is nonempty (Theorem 3.1). A sufficient condition, ensuring that the feasible set is nonempty, is given in Proposition 3.1. Furthermore, in Theorem 3.2, we establish the Karush-Kuhn-Tucker (KKT)-type optimality conditions for (P). Here, to guarantee existence of a Lagrange multiplier for the divergence constraint (1.6), we require an additional assumption (see Assumption 3.1). This assumption is however difficult to check since it also involves the unknown optimal control. Therefore, we approximate the optimal control problem $(\mathrm{P})$ by penalizing the divergence constraint in the following way:

$$
\begin{cases}\min & J(\mathbf{y}, \sigma)+\frac{\tau}{2} \int_{\Omega}|\operatorname{div} \mathbf{y}|^{2} \mathrm{~d} x \\ \text { s.t. } & \operatorname{curl} \mu^{-1} \operatorname{curl} \mathbf{y}-\hat{c} \nabla \operatorname{div} \mathbf{y}+\mathrm{i} \omega \sigma \mathbf{y}=\mathbf{j} \quad \text { in } \Omega \\ & \mathbf{y} \times \mathbf{n}=0 \quad \text { on } \Gamma \\ & 0 \leqslant \sigma(x) \leqslant \sigma_{\max } \quad \text { a.e. in } \Omega\end{cases}
$$

with $\tau>0$. Differently from (P), the KKT-type optimality conditions for the divergence-penalized problem $\left(\mathrm{P}^{\tau}\right)$ can be derived without any additional assumption on the unknown optimal control. Note that every optimal state of $\left(\mathrm{P}^{\tau}\right)$ is not necessarily divergence-free. Nonetheless, due to the presence of the divergence penalization in the objective functional of $\left(\mathrm{P}^{\tau}\right)$, the divergence of every optimal state of $\left(\mathrm{P}^{\tau}\right)$ vanishes as $\tau \rightarrow \infty$. See 
Theorem 4.2 for the convergence of $\left(\mathrm{P}^{\tau}\right)$ towards (P) as $\tau \rightarrow \infty$. Numerical results based on an SQP-algorithm for solving $\left(\mathrm{P}^{\tau}\right)$ are presented in the final section of the paper. Here, the control is discretized by piecewise constant elements, whereas the state is discretized by continuous (vector-valued) $\mathbb{P}_{1}$-elements. We point out that, in the case where the domain $\Omega$ is a convex polyhedron, the use of nodal finite elements for the state discretization is justified by the $\mathbf{H}^{1}$-regularity of the solution to (1.5). In general, this regularity is not true in the case of nonconvex polyhedral domains. This case can be treated using the weighted regularization method introduced by Costabel and Dauge [8].

The mathematical and numerical analysis of Maxwell's equations has become an important research area in applied mathematics. See the monographs [1, 17, 19], the articles $[2-5,7-9,12,13,20]$, and many others. To the best of the author's knowledge, however, only a few studies on the optimal control of Maxwell's equations have been carried out. We refer to the recent results [10, 16, 24, 26, 27, 29] for PDE-constrained optimization involving eddy current equations (with fixed material coefficients) and the article by Hoppe et al. [14] concerning a topology optimization problem for identifying electric conductivity in a two-dimensional $H^{1}(\Omega)$-elliptic system. More recently, Feng et al. [11] have analyzed an identification problem of magnetic permeability and electric permittivity in the time-domain Maxwell equations with BV-regularization. We also mention our previous work [28] on the finite element analysis of optimal control problems in the coefficients of time-harmonic eddy current equations. In that work, however, pointwise constraints on the gradient of the control were considered, leading to $W^{1, \infty}(\Omega)$-regularity in the optimal control. This is rather undesirable as electromagnetic material parameters are typically discontinuous.

We are not aware of any previous study dealing with an optimal control approach based on grad-div regularization and divergence penalization for estimating discontinuous electric conductivity in a 3D bounded domain, that possibly contains non-conducting materials. We emphasize that the estimation for the electric conductivity obtained by solving $(\mathrm{P})$ or $\left(\mathrm{P}^{\tau}\right)$ is allowed to be discontinuous. In fact, no higher regularity property can be derived from the corresponding optimality conditions. The rest of the paper is organized as follows. In next section, we introduce our notation. Section 3 is concerned with the analysis of (P). Then, in Section 4, we investigate $\left(\mathrm{P}^{\tau}\right)$. Finally, an SQP-algorithm for solving $\left(\mathrm{P}^{\tau}\right)$ and various numerical results are presented in Section 5.

\section{Notation}

If $X$ is a Banach space, then we use the notation $\|\cdot\|_{X}$ for a standard norm in $X$. If $X$ a Hilbert space, then $(\cdot, \cdot)_{X}$ denotes the inner product of $X$. A continuous embedding of $X$ in another Banach space $Y$ is denoted by $X \rightarrow Y$. For a complex number $a \in \mathbb{C}^{3}$, its real- and imaginary-parts are denoted by $\operatorname{Re} a$ and $\operatorname{Im} a$, respectively. The space of quadratic Lebesgue integrable real-valued functions on $\Omega$ is denoted by $L^{2}(\Omega)$. On the other hand, $L^{2}(\Omega ; \mathbb{C})$ is the space of quadratic Lebesgue integrable complex-valued functions on $\Omega$. The same notation is used for other Banach spaces to distinguish between complex- and real-valued functions. Furthermore, we use a bold typeface to indicate a Banach space of three-dimensional vector functions. As usual, the Hilbert spaces $\mathbf{H}$ (curl) and $\mathbf{H}$ (div) are defined as follows:

$$
\begin{aligned}
\mathbf{H}(\text { curl }) & :=\left\{\mathbf{q} \in \mathbf{L}^{2}(\Omega ; \mathbb{C}) \mid \operatorname{curl} \mathbf{q} \in \mathbf{L}^{2}(\Omega ; \mathbb{C})\right\} \\
\mathbf{H}(\operatorname{div}) & :=\left\{\mathbf{q} \in \mathbf{L}^{2}(\Omega ; \mathbb{C}) \mid \operatorname{div} \mathbf{q} \in L^{2}(\Omega ; \mathbb{C})\right\}
\end{aligned}
$$

where the curl and div operators are understood in the distributional sense (cf. [2]). Note that $\mathbf{L}^{2}(\Omega ; \mathbb{C}$ ) is endowed with the following inner product:

$$
(\mathbf{q}, \mathbf{v})_{\mathbf{L}^{2}(\Omega ; \mathbb{C})}:=\int_{\Omega} \mathbf{q} \cdot \overline{\mathbf{v}} \mathrm{d} x \quad \forall \mathbf{q}, \mathbf{v} \in \mathbf{L}^{2}(\Omega ; \mathbb{C})
$$

where $\overline{\mathbf{v}}$ denotes the complex conjugate of $\mathbf{v}$. Furthermore, we define

$$
\mathbf{H}_{0}(\text { curl }):=\{\mathbf{q} \in \mathbf{H}(\text { curl }) \mid \mathbf{q} \times \mathbf{n}=0 \text { on } \Gamma\}
$$


and the state space

$$
\mathbf{V}:=\mathbf{H}_{0}(\text { curl }) \cap \mathbf{H}(\text { div })
$$

This space is endowed with the graph norm:

$$
\|\mathbf{v}\|_{\mathbf{v}}=\left(\|\mathbf{v}\|_{\mathbf{L}^{2}(\Omega ; \mathbb{C})}^{2}+\|\operatorname{curl} \mathbf{v}\|_{\mathbf{L}^{2}(\Omega ; \mathbb{C})}^{2}+\|\operatorname{div} \mathbf{v}\|_{L^{2}(\Omega ; \mathbb{C})}^{2}\right)^{1 / 2} \quad \forall \mathbf{v} \in \mathbf{V}
$$

Let us now introduce the sesquilinear form $\alpha_{\sigma}: \mathbf{V} \times \mathbf{V} \rightarrow \mathbb{C}$, defined by

$$
\begin{aligned}
\alpha_{\sigma}(\mathbf{z}, \mathbf{v}):= & \left(\mu^{-1} \operatorname{curl} \mathbf{z}, \text { curl v }\right)_{\mathbf{L}^{2}(\Omega ; \mathbb{C})}+\hat{c}(\operatorname{div} \mathbf{z}, \operatorname{div} \mathbf{v})_{L^{2}(\Omega ; \mathbb{C})} \\
& +(\mathrm{i} \omega \sigma \mathbf{z}, \mathbf{v})_{\mathbf{L}^{2}(\Omega ; \mathbb{C})} \quad \forall \mathbf{z}, \mathbf{v} \in \mathbf{V} .
\end{aligned}
$$

The weak formulation for (1.5) then reads as follows: find $\mathbf{y} \in \mathbf{V}$ such that

$$
\alpha_{\sigma}(\mathbf{y}, \mathbf{v})=(\mathbf{j}, \mathbf{v})_{\mathbf{L}^{2}(\Omega ; \mathbb{C})} \quad \forall \mathbf{v} \in \mathbf{V}
$$

We define the admissible control set and the feasible set associated with (P), respectively, by

$$
\begin{aligned}
\mathcal{U}_{a d} & :=\left\{\sigma \in L^{\infty}(\Omega) \mid 0 \leqslant \sigma(x) \leqslant \sigma_{\max } \text { a.e. in } \Omega\right\} \\
\mathcal{F} & :=\left\{(\mathbf{y}, \sigma) \in \mathbf{V} \times \mathcal{U}_{a d} \mid(\mathbf{y}, \sigma) \text { fulfills (2.1) and } \operatorname{div} \mathbf{y}=0 \text { in } \Omega\right\} .
\end{aligned}
$$

A pair $\left(\mathbf{y}^{\star}, \sigma^{\star}\right) \in \mathcal{F}$ satisfying $J\left(\mathbf{y}^{\star}, \sigma^{\star}\right) \leqslant J(\mathbf{y}, \sigma)$ for all $(\mathbf{y}, \sigma) \in \mathcal{F}$ is called an optimal solution of (P).

\section{Grad-div regularization}

The analysis of the optimal control problem (P) requires the use of the well-known Poincaré-Friedrichs-type inequality:

$$
\|\mathbf{u}\|_{\mathbf{L}^{2}(\Omega)} \leqslant c_{M}\left(\|\operatorname{curl} \mathbf{u}\|_{\mathbf{L}^{2}(\Omega)}+\|\operatorname{div} \mathbf{u}\|_{L^{2}(\Omega)}\right) \quad \forall \mathbf{u} \in \mathbf{V}
$$

with a constant $c_{M}>0$ depending only on the domain $\Omega$. Let us remark that (3.1) follows from a classical contradiction argument using the compactness of the embedding $\mathbf{V} \leftrightarrow \mathbf{L}^{2}(\Omega)$ (see Weck [25]) and the following result:

$$
\mathbf{D F}(\Omega):=\{\mathbf{u} \in \mathbf{V} \mid \operatorname{curl} \mathbf{u}=\mathbf{0}, \operatorname{div} \mathbf{u}=0\}=\{\mathbf{0}\} .
$$

Note that (3.2) holds since $\Gamma$ is connected. If $\Gamma$ were not connected, then the space $\mathbf{D F}(\Omega)$ would contain a nontrivial vector field (see [2, 22]).

Lemma 3.1. For every $\sigma \in L^{3}(\Omega)$, the weak formulation (2.1) admits a unique solution $\mathbf{y}=\mathbf{y}(\sigma) \in \mathbf{V}$. This solution satisfies

$$
\|\mathbf{y}(\sigma)\|_{\mathbf{v}} \leqslant C
$$

with a constant $C>0$ depending only on $\mathbf{j}, \mu_{\max }, \hat{c}$, and $c_{M}$.

Proof. Let $\sigma \in L^{3}(\Omega)$. According to the regularity result by Costabel [7], the embedding

$$
\mathbf{V}=\mathbf{H}_{0}(\text { curl }) \cap \mathbf{H}(\text { div }) \leftrightarrow \mathbf{H}^{1 / 2}(\Omega ; \mathbb{C})
$$

holds true. Then, employing the injection $\mathbf{H}^{1 / 2}(\Omega ; \mathbb{C}) \leftrightarrow \mathbf{L}^{3}(\Omega ; \mathbb{C})$ and (1.2), it follows that

$$
\begin{aligned}
\left|\alpha_{\sigma}(\mathbf{z}, \mathbf{v})\right| \leqslant & \mu_{\min }^{-1}\|\operatorname{curl} \mathbf{z}\|_{\mathbf{L}^{2}(\Omega ; \mathbb{C})}\|\operatorname{curl} \mathbf{v}\|_{\mathbf{L}^{2}(\Omega ; \mathbb{C})}+\hat{c}\|\operatorname{div} \mathbf{z}\|_{L^{2}(\Omega ; \mathbb{C})}\|\operatorname{div} \mathbf{v}\|_{L^{2}(\Omega ; \mathbb{C})} \\
& +\omega\|\sigma\|_{L^{3}(\Omega)}\|\mathbf{z}\|_{\mathbf{L}^{3}(\Omega ; \mathbb{C})}\|\mathbf{v}\|_{\mathbf{L}^{3}(\Omega ; \mathbb{C})} \\
\leqslant & \mu_{\min }^{-1}\|\operatorname{curl} \mathbf{z}\|_{\mathbf{L}^{2}(\Omega ; \mathbb{C})}\|\mathbf{c u r l} \mathbf{v}\|_{\mathbf{L}^{2}(\Omega ; \mathbb{C})}+\hat{c}\|\operatorname{div} \mathbf{z}\|_{L^{2}(\Omega ; \mathbb{C})}\|\operatorname{div} \mathbf{v}\|_{L^{2}(\Omega ; \mathbb{C})} \\
& +c \omega\|\sigma\|_{L^{3}(\Omega)}\|\mathbf{z}\|_{\mathbf{v}}\|\mathbf{v}\|_{\mathbf{v}} \quad \forall \mathbf{z}, \mathbf{v} \in \mathbf{V}
\end{aligned}
$$


with a constant $c>0$ depending only on $\Omega$. In view of (3.5), the sesquilinear form $\alpha_{\sigma}: \mathbf{V} \times \mathbf{V} \rightarrow \mathbb{C}$ is bounded. Taking the real part of $\alpha_{\sigma}(\mathbf{v}, \mathbf{v})$ into account, we now deduce that

$$
\begin{aligned}
\left|\alpha_{\sigma}(\mathbf{v}, \mathbf{v})\right| \geqslant \operatorname{Re}\left(\alpha_{\sigma}(\mathbf{v}, \mathbf{v})\right) & =\int_{\Omega} \mu^{-1}|\operatorname{curl} \mathbf{v}|^{2} \mathrm{~d} x+\hat{c} \int_{\Omega}|\operatorname{div} \mathbf{v}|^{2} \mathrm{~d} x \\
& \geqslant \mu_{\max }^{-1}\|\operatorname{curl} \mathbf{v}\|_{\mathbf{L}^{2}(\Omega ; \mathbb{C})}^{2}+\hat{c}\|\operatorname{div} \mathbf{v}\|_{L^{2}(\Omega ; \mathbb{C})}^{2} \quad \forall \mathbf{v} \in \mathbf{V} .
\end{aligned}
$$

From (3.6) along with Poincaré-Friedrichs-type inequality (3.1), it follows that the sesquilinear form $\alpha_{\sigma}$ : $\mathbf{V} \times \mathbf{V} \rightarrow \mathbb{C}$ is coercive. In conclusion, the Lax-Milgram lemma implies that (2.1) admits a unique solution. Finally, (3.3) follows from (3.6) and the Poincaré-Friedrichs-type inequality (3.1).

Corollary 3.1. Assume that $\Omega$ is convex or of class $\mathrm{e}^{1,1}$. Then, for every $\sigma \in L^{2}(\Omega)$, the weak formulation (2.1) admits a unique solution $\mathbf{y}=\mathbf{y}(\sigma) \in \mathbf{V}$.

Proof. In the case where $\Omega$ is convex or of class $\mathcal{C}^{1,1}$, it is well-known that $\mathbf{V} \rightarrow \mathbf{H}^{1}(\Omega ; \mathbb{C}$ ) holds true (see [2, Proposition 3.7 and Theorem 2.17]). Therefore, as $\mathbf{H}^{1}(\Omega ; \mathbb{C}) \rightarrow \mathbf{L}^{4}(\Omega ; \mathbb{C})$, we can show, analogously to the proof of Lemma 3.1, that the sesquilinear form $\alpha_{\sigma}: \mathbf{V} \times \mathbf{V} \rightarrow \mathbb{C}$, for every $\sigma \in L^{2}(\Omega)$, is well-defined, bounded, and coercive.

In the upcoming proposition, we will make use of the following subspace:

$$
D:=\left\{\varphi \in H_{0}^{1}(\Omega ; \mathbb{C}) \mid \nabla \varphi \in \mathbf{H}(\operatorname{div})\right\} .
$$

Proposition 3.1. Under the assumption that

$$
\operatorname{div} \mathbf{j}=0 \text { in } \Omega
$$

the feasible set $\mathcal{F}$ is nonempty.

Proof. We consider a constant $\sigma_{c} \in\left[0, \sigma_{\max }\right]$, and let $\mathbf{y}$ denote the solution of the weak formulation (2.1) associated with $\sigma \equiv \sigma_{c}$. By definition, every $\varphi \in D$ satisfies $\nabla \varphi \in \mathbf{V}$. Therefore, setting $\mathbf{v}=\nabla \varphi$ with $\varphi \in D$ in (2.1) yields that

$$
\hat{C}(\operatorname{div} \mathbf{y}, \operatorname{div}(\nabla \varphi))_{L^{2}(\Omega ; \mathbb{C})}+\mathrm{i} \omega \sigma_{c}(\mathbf{y}, \nabla \varphi)_{\mathbf{L}^{2}(\Omega ; \mathbb{C})}=(\mathbf{j}, \nabla \varphi)_{\mathbf{L}^{2}(\Omega ; \mathbb{C})} \quad \forall \mathbf{n} \varphi \in D
$$

where we have also used curl $\nabla \equiv 0$. By the distributional definition of the divergence, we obtain from the above variational equality and (3.8) that

$$
\hat{c}(\operatorname{div} \mathbf{y}, \operatorname{div}(\nabla \varphi))_{L^{2}(\Omega ; \mathbb{C})}-\mathbf{i} \omega \sigma_{c}(\operatorname{div} \mathbf{y}, \varphi)_{\mathbf{L}^{2}(\Omega ; \mathbb{C})}=-(\operatorname{div} \mathbf{j}, \varphi)_{\mathbf{L}^{2}(\Omega ; \mathbb{C})}=0 \quad \forall \varphi \in D
$$

which is equivalent to

$$
\left(\operatorname{div} \mathbf{y},-\operatorname{div}(\nabla \varphi)+\mathrm{i} \omega \sigma_{c} \hat{c}^{-1} \varphi\right)_{L^{2}(\Omega ; \mathbb{C})}=0 \quad \forall \varphi \in D .
$$

Now, for any given $u \in L^{2}(\Omega ; \mathbb{C})$, there exists a unique $\varphi \in D$ such that

$$
-\operatorname{div}(\nabla \varphi)+\mathrm{i} \omega \sigma_{c} \hat{c}^{-1} \varphi=u
$$

This follows immediately from the Lax-Milgram lemma along with the distributional definition of the divergence. Therefore, (3.9) implies that

$$
(\operatorname{div} \mathbf{y}, u)_{L^{2}(\Omega ; \mathbb{C})}=0 \quad \forall u \in L^{2}(\Omega ; \mathbb{C})
$$

from which it follows that $\operatorname{div} \mathbf{y}=0$ in $\Omega$. In conclusion, $\mathcal{F} \neq \varnothing$ holds true since $\left(\mathbf{y}, \sigma_{c}\right) \in \mathcal{F}$.

In the following, we assume that $\mathcal{F}$ is nonempty. From Proposition 3.1, we already know that $\mathcal{F} \neq \varnothing$, if $\mathbf{j}$ is divergence-free.

Theorem 3.1. Assume that $\mathcal{F} \neq \varnothing$. Then, the optimal control problem (P) admits at least one optimal solution. 
Proof. Since the functional $J$ is nonnegative and $\mathcal{F} \neq \varnothing$, the infimum $\inf _{(\mathbf{y}, \sigma) \in \mathcal{F}} J(\mathbf{y}, \sigma)$ exists, and there is a minimizing sequence $\left\{\left(\mathbf{y}_{k}, \sigma_{k}\right)\right\}_{k=1}^{\infty} \subset \mathcal{F}$. In other words,

$$
\lim _{k \rightarrow \infty} J\left(\mathbf{y}_{k}, \sigma_{k}\right)=\inf _{(\mathbf{y}, \sigma) \in \mathcal{F}} J(\mathbf{y}, \sigma)
$$

and every $\left(\mathbf{y}_{k}, \sigma_{k}\right)$ satisfies

$$
\begin{aligned}
\left(\mu^{-1} \operatorname{curl} \mathbf{y}_{k}, \operatorname{curl} \mathbf{v}\right)_{\mathbf{L}^{2}(\Omega ; \mathbb{C})}+\left(\mathrm{i} \omega \sigma_{k} \mathbf{y}_{k}, \mathbf{v}\right)_{\mathbf{L}^{2}(\Omega ; \mathbb{C})} & =(\mathbf{j}, \mathbf{v})_{\mathbf{L}^{2}(\Omega ; \mathbb{C})} \quad \forall \mathbf{v} \in \mathbf{V} \\
\operatorname{div} \mathbf{y}_{k} & =0 \text { in } \Omega .
\end{aligned}
$$

Thanks to Lemma 3.1, $\left\{\mathbf{y}_{k}\right\}_{k=1}^{\infty}$ is bounded in $\mathbf{V}$. For this reason and by the structure of $\mathcal{U}_{a d}$, there exists a subsequence of $\left\{\left(\mathbf{y}_{k}, \sigma_{k}\right)\right\}_{k=1}^{\infty}$ denoted again by $\left\{\left(\mathbf{y}_{k}, \sigma_{k}\right)\right\}_{k=1}^{\infty}$ such that

$$
\begin{array}{ll}
\mathbf{y}_{k} \rightarrow \mathbf{y}^{\star} & \text { weakly in } \mathbf{V} \text { as } k \rightarrow \infty \\
\sigma_{k} \rightarrow \sigma^{\star} & \text { weakly in } L^{s}(\Omega) \text { as } k \rightarrow \infty \quad \forall s \in[2, \infty)
\end{array}
$$

for some $\left(\mathbf{y}^{\star}, \sigma^{\star}\right) \in \mathbf{V} \times \mathcal{U}_{a d}$. Let us demonstrate that $\left(\mathbf{y}^{\star}, \sigma^{\star}\right) \in \mathcal{F}$. First, since (3.12) holds for all $k \in \mathbb{N}$, the weak convergence (3.13) yields that $\operatorname{div} \mathbf{y}^{\star}=0$ in $\Omega$. Moreover, in view of (3.4) and the compactness of the injection $\mathbf{H}^{1 / 2}(\Omega ; \mathbb{C}) \rightarrow \mathbf{L}^{2+9}(\Omega ; \mathbb{C})$ for all $\vartheta \in[0,1)$, we infer from (3.13) that

$$
\mathbf{y}_{k} \rightarrow \mathbf{y}^{\star} \quad \text { strongly in } \mathbf{L}^{2+9}(\Omega ; \mathbb{C}) \quad \forall \vartheta \in[0,1) .
$$

This strong convergence and the weak convergence (3.14) yield

$$
\left(\mathrm{i} \omega \sigma_{k} \mathbf{y}_{k}, \mathbf{v}\right)_{\mathbf{L}^{2}(\Omega ; \mathbb{C})} \rightarrow\left(\mathrm{i} \omega \sigma^{\star} \mathbf{y}^{\star}, \mathbf{v}\right)_{\mathbf{L}^{2}(\Omega ; \mathbb{C})} \text { as } k \rightarrow \infty
$$

for every fixed $\mathbf{v} \in \mathbf{V}$. Then, passing to the limit $k \rightarrow \infty$ in (3.11), we obtain from (3.13) and (3.15) that

$$
\left(\mu^{-1} \operatorname{curl}^{\star}{ }^{\star}, \operatorname{curl} \mathbf{v}\right)_{\mathbf{L}^{2}(\Omega ; \mathbb{C})}+\left(\mathbf{i} \omega \sigma^{\star} \mathbf{y}^{\star}, \mathbf{v}\right)_{\mathbf{L}^{2}(\Omega ; \mathbb{C})}=(\mathbf{j}, \mathbf{v})_{\mathbf{L}^{2}(\Omega ; \mathbb{C})} \quad \forall \mathbf{v} \in \mathbf{V} .
$$

Thus, since $\operatorname{div} \mathbf{y}^{\star}=0$ in $\Omega$, it follows that $\left(\mathbf{y}^{\star}, \sigma^{\star}\right) \in \mathcal{F}$. Now, by the lower semicontinuity of the functional $J: \mathbf{L}^{2}(\Omega ; \mathbb{C}) \times L^{2}(\Omega) \rightarrow \mathbb{R}$ and (3.13)-(3.14), we deduce that

$$
J\left(\mathbf{y}^{\star}, \sigma^{\star}\right) \leqslant \liminf _{k \rightarrow \infty} J\left(\mathbf{y}_{k}, \sigma_{k}\right)=\inf _{(\mathbf{y}, \sigma) \in \mathcal{F}} J(\mathbf{y}, \sigma)
$$

from which it follows that $\left(\mathbf{y}^{\star}, \sigma^{\star}\right) \in \mathcal{F}$ is an optimal solution.

\subsection{KKT optimality system}

We denote the control-to-state operator associated with the weak formulation (2.1) by

$$
S: L^{3}(\Omega) \rightarrow \mathbf{V}, \quad \sigma \mapsto \mathbf{y}
$$

that assigns every function $\sigma \in L^{3}(\Omega)$ the unique solution $\mathbf{y} \in \mathbf{V}$ of (2.1). Furthermore, we introduce the operators

$$
\begin{array}{ll}
G_{1}: L^{3}(\Omega) \rightarrow L^{2}(\Omega), & G_{1}(\sigma)=\operatorname{Re}(\operatorname{div}(S(\sigma))) \\
G_{2}: L^{3}(\Omega) \rightarrow L^{2}(\Omega), & G_{2}(\sigma)=\operatorname{Im}(\operatorname{div}(S(\sigma))) .
\end{array}
$$

Employing these operators and the control-to-state operator $S$, the optimal control problem (P) can be reformulated as an optimization problem in Banach spaces:

$$
\left\{\begin{array}{c}
\min _{\sigma \in \mathcal{U}_{a d}} f(\sigma):=J(S(\sigma), \sigma) \\
\text { s.t. } G_{1}(\sigma)=0 \\
G_{2}(\sigma)=0 .
\end{array}\right.
$$

By classical arguments (cf. Tröltzsch [23]), it is standard to derive the Fréchet differentiability of the controlto-state operator with respect to the $L^{\infty}$-topology. 
Lemma 3.2. The control-to-state operator $S: L^{\infty}(\Omega) \rightarrow \mathbf{V}$ is continuously Fréchet-differentiable. For all $\sigma^{\star}, \sigma \in$ $L^{\infty}(\Omega), S^{\prime}\left(\sigma^{\star}\right) \sigma=\mathbf{y}$ is the unique solution of

$$
\int_{\Omega} \mu^{-1} \operatorname{curl} \mathbf{y} \cdot \operatorname{curl} \overline{\mathbf{v}} \mathrm{d} x+\hat{c} \int_{\Omega} \operatorname{div} \mathbf{y} \operatorname{div} \overline{\mathbf{v}} \mathrm{d} x+\int_{\Omega} \mathrm{i} \omega \sigma^{\star} \mathbf{y} \cdot \overline{\mathbf{v}} \mathrm{d} x=-\int_{\Omega} \mathrm{i} \omega \sigma \mathbf{y}^{\star} \cdot \overline{\mathbf{v}} \mathrm{d} x \quad \forall \mathbf{v} \in \mathbf{V}
$$

where $\mathbf{y}^{\star}=S\left(\sigma^{\star}\right)$.

Let us now introduce the Lagrangian functional $\mathcal{L}: L^{3}(\Omega) \times L^{2}(\Omega) \times L^{2}(\Omega) \rightarrow \mathbb{R}$ associated with (P), defined by

$$
\mathcal{L}\left(\sigma, \lambda_{1}, \lambda_{2}\right):=f(\sigma)+\int_{\Omega} \lambda_{1} G_{1}(\sigma) \mathrm{d} x+\int_{\Omega} \lambda_{2} G_{2}(\sigma) \mathrm{d} x .
$$

Lemma 3.3. For all $\sigma^{\star}, \sigma \in L^{\infty}(\Omega)$ and $\lambda_{1}, \lambda_{2} \in L^{2}(\Omega)$, it holds that

$$
\frac{\partial \mathcal{L}}{\partial \sigma}\left(\sigma^{\star}, \lambda_{1}, \lambda_{2}\right) \sigma=-\operatorname{Re}\left(\int_{\Omega} \mathrm{i} \omega \sigma \mathbf{y}^{\star} \cdot \mathbf{p}^{\star} \mathrm{d} x\right)+\varkappa \int_{\Omega} \sigma^{\star} \sigma \mathrm{d} x
$$

where $\mathbf{y}^{\star}=S\left(\sigma^{\star}\right)$, and $\mathbf{p}^{\star} \in \mathbf{V}$ is the solution of

$$
\begin{array}{r}
\int_{\Omega} \mu^{-1} \operatorname{curl} \mathbf{p}^{\star} \cdot \operatorname{curl} \overline{\mathbf{v}} \mathrm{d} x+\hat{c} \int_{\Omega} \operatorname{div} \mathbf{p}^{\star} \operatorname{div} \overline{\mathbf{v}} \mathrm{d} x+\int_{\Omega} \mathrm{i} \omega \sigma^{\star} \mathbf{p}^{\star} \cdot \overline{\mathbf{v}} \mathrm{d} x \\
=\int_{\Omega}\left(\overline{\mathbf{y}^{\star}-\mathbf{y}_{d}}\right) \cdot \overline{\mathbf{v}} \mathrm{d} x+\int_{\Omega}\left(\lambda_{1}-i \lambda_{2}\right) \operatorname{div} \overline{\mathbf{v}} \mathrm{d} x \quad \forall \mathbf{v} \in \mathbf{V} .
\end{array}
$$

Proof. Straightforward computations yield

$$
\begin{aligned}
\frac{\partial \mathcal{L}}{\partial \sigma}\left(\sigma^{\star}, \lambda_{1}, \lambda_{2}\right) \sigma= & \int_{\Omega}\left(\operatorname{Re} \mathbf{y}^{\star}-\operatorname{Re} \mathbf{y}_{d}\right) \cdot \operatorname{Re} \mathbf{y} \mathrm{d} x+\int_{\Omega}\left(\operatorname{Im} \mathbf{y}^{\star}-\operatorname{Im} \mathbf{y}_{d}\right) \cdot \operatorname{Im} \mathbf{y} \mathrm{d} x \\
& +\varkappa \int_{\Omega} \sigma^{\star} \sigma \mathrm{d} x+\int_{\Omega} \lambda_{1} \operatorname{Re}(\operatorname{div} \mathbf{y})+\lambda_{2} \operatorname{Im}(\operatorname{div} \mathbf{y}) \mathrm{d} x
\end{aligned}
$$

where $\mathbf{y}=S^{\prime}\left(\sigma^{\star}\right) \sigma$. Notice that, according to Lemma 3.2, y satisfies

$$
\int_{\Omega} \mu^{-1} \operatorname{curl} \mathbf{y} \cdot \operatorname{curl} \overline{\mathbf{v}} \mathrm{d} x+\hat{c} \int_{\Omega} \operatorname{div} \mathbf{y} \operatorname{div} \overline{\mathbf{v}} \mathrm{d} x+\int_{\Omega} \mathrm{i} \omega \sigma^{\star} \mathbf{y} \cdot \overline{\mathbf{v}} \mathrm{d} x=-\int_{\Omega} \mathrm{i} \omega \sigma \mathbf{y}^{\star} \cdot \overline{\mathbf{v}} \mathrm{d} x \quad \forall \mathbf{v} \in \mathbf{V} .
$$

Let $\mathbf{p}^{\star} \in \mathbf{V}$ denote the solution of (3.18). Setting $\mathbf{v}=\overline{\mathbf{p}}^{\star}$ in (3.20), $\mathbf{v}=\overline{\mathbf{y}}$ in (3.18), and then subtracting the resulting equations, we obtain

$$
\int_{\Omega}\left(\overline{\mathbf{y}^{\star}-\mathbf{y}_{d}}\right) \cdot \mathbf{y} \mathrm{d} x+\int_{\Omega}\left(\lambda_{1}-i \lambda_{2}\right) \operatorname{div} \mathbf{y} \mathrm{d} x=-\int_{\Omega} \mathrm{i} \omega \sigma \mathbf{y}^{\star} \cdot \mathbf{p}^{\star} \mathrm{d} x
$$

and hence

$$
\operatorname{Re}\left(\int_{\Omega}\left(\overline{\mathbf{y}^{\star}-\mathbf{y}_{d}}\right) \cdot \mathbf{y} \mathrm{d} x\right)+\operatorname{Re}\left(\int_{\Omega}\left(\lambda_{1}-i \lambda_{2}\right) \operatorname{div} \mathbf{y} \mathrm{d} x\right)=-\operatorname{Re}\left(\int_{\Omega} \mathrm{i} \omega \sigma \mathbf{y}^{\star} \cdot \mathbf{p}^{\star} \mathrm{d} x\right) .
$$

Since $\operatorname{Re}(\bar{a} b)=\operatorname{Re} a \operatorname{Re} b+\operatorname{Im} a \operatorname{Im} b$ holds for $a, b \in \mathbb{C}$, it follows that

$$
\begin{array}{r}
\int_{\Omega}\left(\operatorname{Re} \mathbf{y}^{\star}-\operatorname{Re} \mathbf{y}_{d}\right) \cdot \operatorname{Re} \mathbf{y} \mathrm{d} x+\int_{\Omega}\left(\operatorname{Im} \mathbf{y}^{\star}-\operatorname{Im} \mathbf{y}_{d}\right) \cdot \operatorname{Im} \mathbf{y} \mathrm{d} x \\
+\int_{\Omega} \lambda_{1} \operatorname{Re}(\operatorname{div} \mathbf{y}) \mathrm{d} x+\int_{\Omega} \lambda_{2} \operatorname{Im}(\operatorname{div} \mathbf{y}) \mathrm{d} x=-\operatorname{Re}\left(\int_{\Omega} \mathrm{i} \omega \sigma \mathbf{y}^{\star} \cdot \mathbf{p}^{\star} \mathrm{d} x\right) .
\end{array}
$$

Inserting this identity in (3.19) results in the formula (3.17). 
Definition 3.1. Let $\left(\mathbf{y}^{\star}, \sigma^{\star}\right) \in \mathcal{F}$ be an optimal solution of $(\mathrm{P})$. Then $\left(\lambda_{1}^{\star}, \lambda_{2}^{\star}\right) \in L^{2}(\Omega) \times L^{2}(\Omega)$ is called a pair of Lagrange multipliers associated with $\left(\mathbf{y}^{\star}, \sigma^{\star}\right)$, if it satisfies

$$
\frac{\partial \mathcal{L}}{\partial \sigma}\left(\sigma^{\star}, \lambda_{1}^{\star}, \lambda_{2}^{\star}\right)\left(\sigma-\sigma^{\star}\right) \geqslant 0 \quad \forall \sigma \in \mathcal{U}_{a d} .
$$

In what follows, let $\left(\mathbf{y}^{\star}, \sigma^{\star}\right) \in \mathcal{F}$ be an optimal solution of (P). To derive existence of a pair of Lagrange multipliers associated with $\left(\mathbf{y}^{\star}, \sigma^{\star}\right)$, we invoke the well-known constraint qualification by Zowe and Kurcyusz [30] (cf. also [23, p. 331]), which requires the use of the following cone:

$$
\mathcal{C}\left(\sigma^{\star}\right)=\left\{y\left(\sigma-\sigma^{\star}\right) \mid y \geqslant 0, \sigma \in \mathcal{U}_{a d}\right\} .
$$

Assumption 3.1. Assume that, for every $\varphi \in D$ (see (3.7) for the definition of $D$ ), there exists a function $\sigma \in$ $\mathcal{C}\left(\sigma^{\star}\right)$ such that

$$
S^{\prime}\left(\sigma^{\star}\right) \sigma=\nabla \varphi .
$$

According to Lemma 3.2, this is equivalent to

$$
\hat{c} \int_{\Omega} \operatorname{div}(\nabla \varphi) \operatorname{div} \overline{\mathbf{v}} \mathrm{d} x+\int_{\Omega} \mathrm{i} \omega \sigma^{\star} \nabla \varphi \cdot \overline{\mathbf{v}} \mathrm{d} x=-\int_{\Omega} \mathrm{i} \omega \sigma \mathbf{y}^{\star} \cdot \overline{\mathbf{v}} \mathrm{d} x \quad \forall \mathbf{v} \in \mathbf{V}
$$

since $\operatorname{curl} \nabla \equiv 0$.

Lemma 3.4. Suppose that $\sigma^{\star}$ fulfills Assumption 3.1. Then,

$$
G_{1}^{\prime}\left(\sigma^{\star}\right) \mathcal{C}\left(\sigma^{\star}\right)=L^{2}(\Omega), \quad G_{2}^{\prime}\left(\sigma^{\star}\right) \mathcal{C}\left(\sigma^{\star}\right)=L^{2}(\Omega) .
$$

Proof. Let $u \in L^{2}(\Omega ; \mathbb{C})$, and let $\varphi \in H_{0}^{1}(\Omega ; \mathbb{C})$ be the solution of

$$
-(\nabla \varphi, \nabla \xi)=(u, \xi) \quad \forall \xi \in H_{0}^{1}(\Omega ; \mathbb{C}) .
$$

The distributional definition of the divergence yields that

$$
\operatorname{div}(\nabla \varphi)=u \in L^{2}(\Omega ; \mathbb{C}) \quad \Longrightarrow \quad \nabla \varphi \in \mathbf{H}(\text { div }) \quad \Longrightarrow \quad \varphi \in D .
$$

Now, Assumption 3.1 implies the existence of a function $\sigma \in \mathcal{C}\left(\sigma^{\star}\right)$ such that

$$
S^{\prime}\left(\sigma^{\star}\right) \sigma=\nabla \varphi .
$$

Then, we obtain from (3.22)-(3.23) that

$$
\begin{aligned}
& G_{1}^{\prime}\left(\sigma^{\star}\right) \sigma=\operatorname{Re}\left(\operatorname{div}\left(S^{\prime}\left(\sigma^{\star}\right) \sigma\right)\right)=\operatorname{Re}(\operatorname{div}(\nabla \varphi))=\operatorname{Re} u \\
& G_{2}^{\prime}\left(\sigma^{\star}\right) \sigma=\operatorname{Im}\left(\operatorname{div}\left(S^{\prime}\left(\sigma^{\star}\right) \sigma\right)\right)=\operatorname{Im}(\operatorname{div}(\nabla \varphi))=\operatorname{Im} u .
\end{aligned}
$$

As $u \in L^{2}(\Omega$; $\mathbb{C})$ was chosen arbitrarily, we conclude that (3.21) is valid.

Theorem 3.2. Let $\left(\mathbf{y}^{\star}, \sigma^{\star}\right) \in \mathcal{F}$ be an optimal solution of (P). Suppose that the optimal control $\sigma^{\star}$ fulfills Assumption 3.1. Then there exist $\left(\lambda_{1}^{\star}, \lambda_{2}^{*}\right) \in L^{2}(\Omega) \times L^{2}(\Omega)$ and $\mathbf{p}^{\star} \in \mathbf{V}$ such that

$$
\begin{array}{r}
\int_{\Omega} \mu^{-1} \operatorname{curl}^{\star} \cdot \operatorname{curl} \overline{\mathbf{v}} \mathrm{d} x+\int_{\Omega} \mathrm{i} \omega \sigma^{\star} \mathbf{y}^{\star} \cdot \overline{\mathbf{v}} \mathrm{d} x=\int_{\Omega} \mathbf{j} \cdot \overline{\mathbf{v}} \mathrm{d} x \quad \forall \mathbf{v} \in \mathbf{V} \\
\int_{\Omega} \mu^{-1} \operatorname{curl}^{\star} \cdot \operatorname{curl} \overline{\mathbf{v}} \mathrm{d} x+\hat{c} \int_{\Omega} \operatorname{div} \mathbf{p}^{\star} \operatorname{div} \overline{\mathbf{v}} \mathrm{d} x+\int_{\Omega} \mathrm{i} \omega \sigma^{\star} \mathbf{p}^{\star} \cdot \overline{\mathbf{v}} \mathrm{d} x \\
=\int_{\Omega}\left(\overline{\mathbf{y}^{\star}-\mathbf{y}_{d}}\right) \cdot \overline{\mathbf{v}} \mathrm{d} x+\int_{\Omega}\left(\lambda_{1}^{\star}-i \lambda_{2}^{\star}\right) \operatorname{div} \overline{\mathbf{v}} \mathrm{d} x \quad \forall \mathbf{v} \in \mathbf{V} \\
\sigma^{\star}(x)=\mathbb{P}_{\left[0, \sigma_{\max }\right]}\left(\frac{\omega}{\varkappa} \operatorname{Re}\left[i \mathbf{y}^{\star}(x) \cdot \mathbf{p}^{\star}(x)\right]\right) \text { a.e. in } \Omega
\end{array}
$$

where the projection $\mathbb{P}_{\left[0, \sigma_{\max }\right]}: \mathbb{R} \rightarrow\left[0, \sigma_{\max }\right]$ is defined by

$$
\mathbb{P}_{\left[0, \sigma_{\max }\right]}(\delta)=\max \left\{0, \min \left\{\delta, \sigma_{\max }\right\}\right\} \quad \forall \delta \in \mathbb{R} .
$$


Proof. As shown in Lemma 3.4, the constraint qualification (3.21) holds true. For this reason, there exists a pair of Lagrange multipliers $\left(\lambda_{1}^{\star}, \lambda_{2}^{\star}\right) \in L^{2}(\Omega) \times L^{2}(\Omega)$ satisfying

$$
\frac{\partial \mathcal{L}}{\partial \sigma}\left(\sigma^{\star}, \lambda_{1}^{\star}, \lambda_{2}^{\star}\right)\left(\sigma-\sigma^{\star}\right) \geqslant 0 \quad \forall \sigma \in \mathcal{U}_{a d} .
$$

In view of Lemma 3.3, we have

$$
\frac{\partial \mathcal{L}}{\partial \sigma}\left(\sigma^{\star}, \lambda_{1}^{\star}, \lambda_{2}^{\star}\right)\left(\sigma-\sigma^{\star}\right)=-\operatorname{Re}\left(\int_{\Omega} \mathrm{i} \omega\left(\sigma-\sigma^{\star}\right) \mathbf{y}^{\star} \cdot \mathbf{p}^{\star} \mathrm{d} x\right)+\varkappa \int_{\Omega} \sigma^{\star}\left(\sigma-\sigma^{\star}\right) \mathrm{d} x \quad \forall \sigma \in \mathcal{U}_{a d}
$$

where $\mathbf{p}^{\star} \in \mathbf{V}$ is the unique solution of (3.25). Thus (3.27) is equivalent to

$$
\int_{\Omega}\left(\sigma-\sigma^{\star}\right)\left(\sigma^{\star}-\frac{\omega}{\varkappa} \operatorname{Re}\left[i \mathbf{y}^{\star} \cdot \mathbf{p}^{\star}\right]\right) \mathrm{d} x \geqslant 0 \quad \forall \sigma \in \mathcal{U}_{a d} .
$$

A standard pointwise evaluation of the above variational inequality leads to the projection formula (3.26). In conclusion, the assertion is valid.

Remark 3.1. As $\mathbf{p}^{\star}$ and $\mathbf{y}^{\star}$ are of class $\mathbf{V} \leftrightarrow \mathbf{H}^{1 / 2}(\Omega ; \mathbb{C})$, the product $\mathbf{y}^{\star} \cdot \mathbf{p}^{\star}$ is only well-defined as an element of $L^{3 / 2}(\Omega ; \mathbb{C})$. For this reason, we cannot extract any higher regularity property from the projection formula (3.26). In conclusion, every optimal control $\sigma^{\star}$ of (P), which serves as an approximation for the electric conductivity of $\Omega$, belongs only to $L^{\infty}(\Omega)$ and is allowed to be highly discontinuous.

It should be emphasized that Assumption 3.1 involves the unknown optimal control $\sigma^{\star}$. We do not know how to check this assumption in practice. Therefore, in next section, we consider the divergence-penalized problem $\left(\mathrm{P}^{\tau}\right)$, where no additional assumption is required for deriving the corresponding KKT-type optimality conditions.

\section{Divergence penalization}

This section is devoted to the divergence-penalized problem

$$
\left\{\begin{array}{cc}
\min _{(\mathbf{y}, \sigma) \in \mathbf{V} \times \mathcal{U}_{a d}} J_{\tau}(\mathbf{y}, \sigma):=J(\mathbf{y}, \sigma)+\frac{\tau}{2} \int_{\Omega}|\operatorname{div} \mathbf{y}|^{2} \mathrm{~d} x \\
\text { s.t. } \quad \alpha_{\sigma}(\mathbf{y}, \mathbf{v})=(\mathbf{j}, \mathbf{v})_{\mathbf{L}^{2}(\Omega ; \mathbb{C})} \quad \forall \mathbf{v} \in \mathbf{V}
\end{array}\right.
$$

where the violation of the eliminated divergence constraint is minimized by the penalty functional $I_{\tau}$ : $\mathbf{H}($ div $) \rightarrow \mathbb{R}, I_{\tau}(\mathbf{y})=\frac{1}{2} \tau \int_{\Omega}|\operatorname{div} \mathbf{y}|^{2} \mathrm{~d} x$. Note that every pair $(S(\sigma), \sigma)$, with $\sigma \in \mathcal{U}_{a d}$, is feasible for $\left(\mathrm{P}^{\tau}\right)$ for all $\tau>0$.

Lemma 4.1. For every $\tau>0$, the divergence-penalized problem $\left(\mathrm{P}^{\tau}\right)$ admits at least one optimal solution $\left(\mathbf{y}_{\tau}, \sigma_{\tau}\right) \in \mathbf{V} \times \mathcal{U}_{\text {ad }}$. If, for a fixed $\tau_{1}>0$, there is an optimal solution $\left(\mathbf{y}_{\tau_{1}}, \sigma_{\tau_{1}}\right)$ of $\left(\mathrm{P}_{\tau_{1}}\right)$ satisfying $\operatorname{div} \mathbf{y}_{\tau_{1}}=0$ in $\Omega$, then $\left(\mathbf{y}_{\tau_{1}}, \sigma_{\tau_{1}}\right)$ is an optimal solution of $(\mathrm{P})$ and $\left(\mathrm{P}^{\tau}\right)$ for all $\tau>\tau_{1}$.

Proof. The existence result can be justified analogously as in the proof of Theorem 3.1. Now, suppose that $\left(\mathbf{y}_{\tau_{1}}, \sigma_{\tau_{1}}\right) \in \mathbf{V} \times \mathcal{U}_{a d}$ is an optimal solution of $\left(\mathrm{P}_{\tau_{1}}\right)$ satisfying

$$
\operatorname{div} \mathbf{y}_{\tau_{1}}=0 \text { in } \Omega
$$

which in particular yields that $\left(\mathbf{y}_{\tau_{1}}, \sigma_{\tau_{1}}\right) \in \mathcal{F}$. Since every element $(\mathbf{y}, \sigma) \in \mathcal{F}$ is feasible for $\left(\mathrm{P}_{\tau_{1}}\right),(4.1)$ gives

$$
J\left(\mathbf{y}_{\tau_{1}}, \sigma_{\tau_{1}}\right)=J_{\tau_{1}}\left(\mathbf{y}_{\tau_{1}}, \sigma_{\tau_{1}}\right) \leqslant J_{\tau_{1}}(\mathbf{y}, \sigma)=J(\mathbf{y}, \sigma) \quad \forall(\mathbf{y}, \sigma) \in \mathcal{F} .
$$

It follows therefore that $\left(\mathbf{y}_{\tau_{1}}, \sigma_{\tau_{1}}\right)$ is an optimal solution of $(\mathrm{P})$. Let $\left(\mathbf{y}_{\tau}, \sigma_{\tau}\right) \in \mathbf{V} \times \mathcal{U}_{a d}$ be an optimal solution of $\left(\mathrm{P}^{\tau}\right)$ for any fixed $\tau>\tau_{1}$. Using again (4.1) and since $\left(\mathbf{y}_{\tau}, \sigma_{\tau}\right)$ is feasible for $\left(\mathrm{P}_{\tau_{1}}\right)$, we have that

$$
J_{\tau}\left(\mathbf{y}_{\tau_{1}}, \sigma_{\tau_{1}}\right)=J_{\tau_{1}}\left(\mathbf{y}_{\tau_{1}}, \sigma_{\tau_{1}}\right) \leqslant J_{\tau_{1}}\left(\mathbf{y}_{\tau}, \sigma_{\tau}\right) \leqslant J_{\tau}\left(\mathbf{y}_{\tau}, \sigma_{\tau}\right) .
$$

For this reason, we conclude that $\left(\mathbf{y}_{\tau_{1}}, \sigma_{\tau_{1}}\right)$ is an optimal solution of $\left(\mathrm{P}^{\tau}\right)$. This completes the proof. 
In the following theorem, we present the KKT-type optimality conditions for $\left(\mathrm{P}^{\tau}\right)$. In contrast to $(\mathrm{P})$, the optimality conditions for $\left(\mathrm{P}^{\tau}\right)$ can be established without any additional assumption on the optimal solution.

Theorem 4.1. Let $\tau>0$ and $\left(\mathbf{y}_{\tau}, \sigma_{\tau}\right) \in \mathbf{V} \times \mathfrak{U}_{\text {ad }}$ be an optimal solution of $\left(\mathrm{P}^{\tau}\right)$. Then, there exists a unique $\mathbf{p}_{\tau} \in \mathbf{V}$ such that

$$
\begin{array}{r}
\int_{\Omega} \mu^{-1} \operatorname{curl} \mathbf{y}_{\tau} \cdot \operatorname{curl} \overline{\mathbf{v}} \mathrm{d} x+\hat{c} \int_{\Omega} \operatorname{div} \mathbf{y}_{\tau} \operatorname{div} \overline{\mathbf{v}} \mathrm{d} x+\int_{\Omega} \mathrm{i} \omega \sigma_{\tau} \mathbf{y}_{\tau} \cdot \overline{\mathbf{v}} \mathrm{d} x=\int_{\Omega} \mathbf{j} \cdot \overline{\mathbf{v}} \mathrm{d} x \quad \forall \mathbf{v} \in \mathbf{V} \\
\int_{\Omega} \mu^{-1} \operatorname{curl} \mathbf{p}_{\tau} \cdot \operatorname{curl} \overline{\mathbf{v}} \mathrm{d} x+\hat{c} \int_{\Omega} \operatorname{div} \mathbf{p}_{\tau} \operatorname{div} \overline{\mathbf{v}} \mathrm{d} x+\int_{\Omega} \mathrm{i} \omega \sigma_{\tau} \mathbf{p}_{\tau} \cdot \overline{\mathbf{v}} \mathrm{d} x \\
=\int_{\Omega}\left(\overline{\mathbf{y}_{\tau}-\mathbf{y}_{d}}\right) \cdot \overline{\mathbf{v}} \mathrm{d} x+\tau \int_{\Omega} \operatorname{div} \overline{\mathbf{y}}_{\tau} \operatorname{div} \overline{\mathbf{v}} \mathrm{d} x \quad \forall \mathbf{v} \in \mathbf{V} \\
\sigma_{\tau}(x)=\mathbb{P}_{\left[0, \sigma_{\max }\right]}\left(\frac{\omega}{\varkappa} \operatorname{Re}\left[i \mathbf{y}_{\tau}(x) \cdot \mathbf{p}_{\tau}(x)\right]\right) \text { a.e. in } \Omega .
\end{array}
$$

Proof. Employing the control-to-state operator, $\left(\mathrm{P}^{\tau}\right)$ can be written in the following form:

$$
\min _{\sigma \in \mathcal{U}_{a d}} f_{\tau}(\sigma):=J_{\tau}(S(\sigma), \sigma) .
$$

Since $U_{a d}$ is a convex subset of $L^{\infty}(\Omega)$, and $f_{\tau}: L^{\infty}(\Omega) \rightarrow \mathbb{R}$ is Fréchet-differentiable, the optimal control $\sigma_{\tau}$ satisfies the following variational inequality:

$$
f_{\tau}^{\prime}\left(\sigma_{\tau}\right)\left(\sigma-\sigma_{\tau}\right) \geqslant 0 \quad \forall \sigma \in \mathcal{U}_{a d} .
$$

Analogously as in the proof of Lemma 3.3, it holds that

$$
f_{\tau}^{\prime}\left(\sigma_{\tau}\right) \sigma=-\operatorname{Re}\left(\int_{\Omega} \mathrm{i} \omega \sigma \mathbf{y}_{\tau} \cdot \mathbf{p}_{\tau} \mathrm{d} x\right)+\varkappa \int_{\Omega} \sigma_{\tau} \sigma \mathrm{d} x
$$

where $\mathbf{p}_{\tau}$ is the unique solution of (4.3). Using the above identity, we can show that (4.5) is equivalent to the optimality system (4.2)-(4.4).

Remark 4.1. From the projection formula (4.4), we cannot extract any higher regularity property for the optimal control $\sigma_{\tau}$.

Theorem 4.2. Let $\left\{\left(\mathbf{y}_{\tau}, \sigma_{\tau}\right)\right\}_{\tau>0} \subset \mathbf{V} \times \mathcal{U}_{\text {ad }}$ be a sequence of optimal solutions of $\left(\mathrm{P}^{\tau}\right)$. Then

$$
\operatorname{div} \mathbf{y}_{\tau} \rightarrow 0 \quad \text { strongly in } L^{2}(\Omega ; \mathbb{C}) \text { as } \tau \rightarrow \infty .
$$

There is a subsequence of $\left\{\left(\mathbf{y}_{\tau}, \sigma_{\tau}\right)\right\}_{\tau>0}$, denoted by $\left\{\left(\mathbf{y}_{\tau}, \sigma_{\tau}\right)\right\}_{\tau>0}$, such that

$$
\begin{aligned}
& \mathbf{y}_{\tau} \rightarrow \mathbf{y}^{\star} \quad \text { strongly in } \mathbf{L}^{2}(\Omega ; \mathbb{C}) \text { as } \tau \rightarrow \infty
\end{aligned}
$$

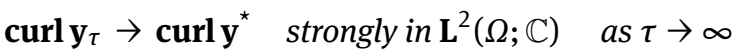

$$
\begin{aligned}
& \sigma_{\tau} \rightarrow \sigma^{\star} \quad \text { strongly in } L^{S}(\Omega) \quad \text { as } \tau \rightarrow \infty \quad \forall s \in[1, \infty)
\end{aligned}
$$

where $\left(\mathbf{y}^{\star}, \sigma^{\star}\right) \in \mathcal{F}$ is an optimal solution of $(\mathrm{P})$. Every weakly converging subsequence of the sequence $\left\{\left(\mathbf{y}_{\tau}, \sigma_{\tau}\right)\right\}_{\tau>0}$ converges strongly in the $\mathbf{V} \times L^{S}(\Omega)$-topology, for all $s \in[1, \infty)$, towards an optimal solution of $(\mathrm{P})$.

Proof. In view of Lemma 3.1, $\left\{\mathbf{y}_{\tau}\right\}_{\tau>0}$ is bounded in $\mathbf{V}$. For this reason and by the structure of $\mathcal{U}_{a d}$, we can extract a subsequence of $\left\{\left(\mathbf{y}_{\tau}, \sigma_{\tau}\right)\right\}_{\tau>0}$, denoted again by $\left\{\left(\mathbf{y}_{\tau}, \sigma_{\tau}\right)\right\}_{\tau>0}$, such that

$$
\begin{array}{ll}
\mathbf{y}_{\tau} \rightarrow \mathbf{y}^{\star} & \text { weakly in } \mathbf{V} \quad \text { as } \tau \rightarrow \infty \\
\sigma_{\tau} \rightarrow \sigma^{\star} & \text { weakly in } L^{S}(\Omega) \quad \text { as } \tau \rightarrow \infty \quad \forall s \in[2, \infty)
\end{array}
$$


for some $\left(\mathbf{y}^{\star}, \sigma^{\star}\right) \in \mathbf{V} \times \mathfrak{U}_{a d}$. Notice that the weak convergence (4.9) implies in particular that

$$
\operatorname{div} \mathbf{y} \tau \rightarrow \operatorname{div} \mathbf{y}^{\star} \quad \text { weakly in } L^{2}(\Omega ; \mathbb{C}) \quad \text { as } \tau \rightarrow \infty .
$$

Using (4.9) and (4.10), we can show, analogously as in the proof of Theorem 3.1, that the weak limit $\left(\mathbf{y}^{\star}, \sigma^{\star}\right)$ satisfies

$$
\left(\mu^{-1} \operatorname{curl}^{\star} \mathbf{y}^{\star}, \operatorname{curl} \mathbf{v}\right)_{\mathbf{L}^{2}(\Omega ; \mathbb{C})}+\hat{c}\left(\operatorname{div} \mathbf{y}^{\star}, \operatorname{div} \mathbf{v}\right)_{L^{2}(\Omega ; \mathbb{C})}+\left(\mathbf{i} \omega \sigma^{\star} \mathbf{y}^{\star}, \mathbf{v}\right)_{\mathbf{L}^{2}(\Omega ; \mathbb{C})}=(\mathbf{j}, \mathbf{v})_{\mathbf{L}^{2}(\Omega ; \mathbb{C})} \quad \forall \mathbf{v} \in \mathbf{V} .
$$

Let $(\widetilde{\mathbf{y}}, \widetilde{\sigma}) \in \mathcal{F}$ be an optimal solution of $(\mathrm{P})$. As $(\widetilde{\mathbf{y}}, \widetilde{\sigma})$ is feasible for $\left(\mathrm{P}^{\tau}\right)$ for all $\tau>0$, we have

$$
J\left(\mathbf{y}_{\tau}, \sigma_{\tau}\right)+\frac{\tau}{2}\left\|\operatorname{div} \mathbf{y}_{\tau}\right\|_{L^{2}(\Omega ; \mathbb{C})}^{2}=J_{\tau}\left(\mathbf{y}_{\tau}, \sigma_{\tau}\right) \leqslant J_{\tau}(\widetilde{\mathbf{y}}, \widetilde{\sigma}) \underbrace{=}_{\operatorname{div} \widetilde{\mathbf{y}}=0} J(\widetilde{\mathbf{y}}, \widetilde{\sigma}) \quad \forall \tau>0 .
$$

Since $J$ is nonnegative, it follows from (4.13) that

$$
\operatorname{div} \mathbf{y}_{\tau} \rightarrow 0 \quad \text { strongly in } L^{2}(\Omega ; \mathbb{C}) \quad \text { as } \tau \rightarrow \infty .
$$

This convergence and (4.11) yield

$$
\operatorname{div} \mathbf{y}^{\star}=0 \text { in } \Omega .
$$

Furthermore, using the weak lower semicontinuity of $J: \mathbf{L}^{2}(\Omega ; \mathbb{C}) \times L^{2}(\Omega) \rightarrow \mathbb{R}$ along with the weak converges (4.9)-(4.10), we infer that

$$
J\left(\mathbf{y}^{\star}, \sigma^{\star}\right) \leqslant \liminf _{\tau \rightarrow \infty} J\left(\mathbf{y}_{\tau}, \sigma_{\tau}\right) \leqslant \limsup _{\tau \rightarrow \infty} J\left(\mathbf{y}_{\tau}, \sigma_{\tau}\right) \underbrace{\leqslant}_{(4.13)} J(\widetilde{\mathbf{y}}, \widetilde{\sigma}) .
$$

According to (4.12) and (4.15), $\left(\mathbf{y}^{\star}, \sigma^{\star}\right) \in \mathcal{F}$. Thus, since $(\widetilde{\mathbf{y}}, \widetilde{\sigma})$ is an optimal solution of (P), we conclude from (4.16) that $\left(\mathbf{y}^{\star}, \sigma^{\star}\right)$ is also an optimal solution of (P). In particular,

$$
J\left(\mathbf{y}^{\star}, \sigma^{\star}\right)=J(\widetilde{\mathbf{y}}, \widetilde{\sigma}) .
$$

Let us now prove (4.6), (4.7), and (4.8). In view of the compactness of the injection $\mathbf{V} \rightarrow \mathbf{L}^{2+9}(\Omega$; $\mathbb{C}$ ) for all $\vartheta \in[0,1)$ (see (3.4)), we obtain from (4.9) that

$$
\mathbf{y}_{\tau} \rightarrow \mathbf{y}^{\star} \quad \text { strongly in } \mathbf{L}^{2+\vartheta}(\Omega ; \mathbb{C}) \quad \forall \vartheta \in[0,1) \quad \text { as } \tau \rightarrow \infty .
$$

From (4.16) and (4.17), it follows that $\lim _{\tau \rightarrow \infty} J\left(\mathbf{y}_{\tau}, \sigma_{\tau}\right)=J\left(\mathbf{y}^{\star}, \sigma^{\star}\right)$, or equivalently

$$
\lim _{\tau \rightarrow \infty}\left(\frac{1}{2}\left\|\mathbf{y}_{\tau}-\mathbf{y}_{d}\right\|_{\mathbf{L}^{2}(\Omega ; \mathbb{C})}^{2}+\frac{\varkappa}{2}\left\|\sigma_{\tau}\right\|_{L^{2}(\Omega)}^{2}\right)=\frac{1}{2}\left\|\mathbf{y}^{\star}-\mathbf{y}_{d}\right\|_{\mathbf{L}^{2}(\Omega ; \mathbb{C})}^{2}+\frac{\varkappa}{2}\left\|\sigma^{\star}\right\|_{L^{2}(\Omega)}^{2} .
$$

Thus, (4.18) implies that $\lim _{\tau \rightarrow \infty}\left\|\sigma_{\tau}\right\|_{L^{2}(\Omega)}=\left\|\sigma^{\star}\right\|_{L^{2}(\Omega)}$ and hence, along with the weak convergence (4.10), we obtain

$$
\sigma_{\tau} \rightarrow \sigma^{\star} \quad \text { strongly in } L^{2}(\Omega) \quad \text { as } \tau \rightarrow \infty .
$$

Since $\sigma^{\star}, \sigma_{\tau} \in \mathcal{U}_{\text {ad }}$ for all $\tau>0$,

$$
\begin{aligned}
\int_{\Omega}\left|\sigma_{\tau}-\sigma^{\star}\right|^{s} \mathrm{~d} x & =\int_{\Omega}\left|\sigma_{\tau}-\sigma^{\star}\right|^{S-2}\left|\sigma_{\tau}-\sigma^{\star}\right|^{2} \mathrm{~d} x \\
& \leqslant \int_{\Omega} \sigma_{\max }^{s-2}\left|\sigma_{\tau}-\sigma^{\star}\right|^{2} \mathrm{~d} x=\sigma_{\max }^{s-2}\left\|\sigma_{\tau}-\sigma^{\star}\right\|_{L^{2}(\Omega)}^{2} \quad \forall s \in(2, \infty) .
\end{aligned}
$$

Then, passing to the limit $\tau \rightarrow \infty$, (4.19) yields

$$
\sigma_{\tau} \rightarrow \sigma^{\star} \quad \text { strongly in } L^{s}(\Omega) \quad \text { as } \tau \rightarrow \infty \quad \forall s \in[2, \infty) .
$$


It remains to prove (4.7). By definition, every $\mathbf{y}_{\tau}$ satisfies

$$
\left(\mu^{-1} \operatorname{curl} \mathbf{y}_{\tau}, \operatorname{curl} \mathbf{y}_{\tau}\right)_{\mathbf{L}^{2}(\Omega ; \mathbb{C})}=-\hat{c}\left(\operatorname{div} \mathbf{y}_{\tau}, \operatorname{div} \mathbf{y}_{\tau}\right)_{\mathbf{L}^{2}(\Omega ; \mathbb{C})}-\left(\mathbf{i} \omega \sigma_{y} \mathbf{y}_{\tau}, \mathbf{y}_{\tau}\right)_{\mathbf{L}^{2}(\Omega ; \mathbb{C})}+\left(\mathbf{j}, \mathbf{y}_{\tau}\right)_{\mathbf{L}^{2}(\Omega ; \mathbb{C})}
$$

Passing to the limit $\tau \rightarrow \infty$, (4.14), (4.18), and (4.20) imply

$$
\left(\mu^{-1} \operatorname{curl}_{\tau}, \operatorname{curl} \mathbf{y}_{\tau}\right)_{\mathbf{L}^{2}(\Omega ; \mathbb{C})} \rightarrow \underbrace{-\left(\mathbf{i} \omega \sigma^{\star} \mathbf{y}^{\star}, \mathbf{y}^{\star}\right)_{\mathbf{L}^{2}(\Omega ; \mathbb{C})}+\left(\mathbf{j}, \mathbf{y}^{\star}\right)_{\mathbf{L}^{2}(\Omega ; \mathbb{C})}}_{=\left(\mu^{-1} \operatorname{curl}^{\star}, \operatorname{curl}^{\star}\right)_{\mathbf{L}^{2}(\Omega ; \mathbb{C})} \text { by }(4.12) \text { and }(4.15)} \text { as } \tau \rightarrow \infty \text {. }
$$

In other words

$$
\left\|\mu^{-1 / 2} \operatorname{curl} \mathbf{y}_{\tau}\right\|_{\mathbf{L}^{2}(\Omega ; \mathbb{C})}^{2} \rightarrow\left\|\mu^{-1 / 2} \operatorname{curl}^{\mathbf{y}^{*}}\right\|_{\mathbf{L}^{2}(\Omega ; \mathbb{C})}^{2} \text { as } \tau \rightarrow \infty .
$$

Further, since $\mu^{-1 / 2} \in L^{\infty}(\Omega)$, the weak convergence (4.9) implies that

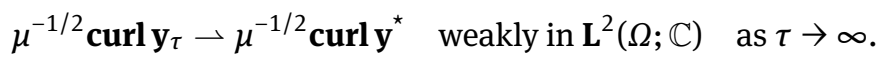

This weak convergence together with (4.21) gives the strong convergence

$$
\mu^{-1 / 2} \text { curl } \mathbf{y}_{\tau} \rightarrow \mu^{-1 / 2} \text { curl }^{\star} \quad \text { strongly in } \mathbf{L}^{2}(\Omega ; \mathbb{C}) \quad \text { as } \tau \rightarrow \infty \text {. }
$$

In conclusion, (4.7) is valid. This completes the proof.

Corollary 4.1. Let $\left\{\left(\mathbf{y}_{\tau}, \sigma_{\tau}\right)\right\}_{\tau>0} \subset \mathbf{V} \times \mathcal{U}_{a d}$ be a sequence of optimal solutions of ( $\left.\mathrm{P}^{\tau}\right)$. If (P) admits a unique solution, then $\left\{\left(\mathbf{y}_{\tau}, \sigma_{\tau}\right)\right\}_{\tau>0}$ converges strongly in the $\mathbf{V} \times L^{S}(\Omega)$-topology, for all $s \in[1, \infty)$, towards the optimal solution of $(\mathrm{P})$.

\section{Numerical experiment}

We consider an SQP-algorithm for the numerical solution of $\left(\mathrm{P}^{\tau}\right)$. Let us first point out that $(\mathrm{P})$ can be equivalently formulated as a minimization problem involving only real-valued quantities. More precisely, we denote the real-part and the imaginary-part of the state $\mathbf{y}$, respectively, by $\mathbf{y}_{1}$ and $\mathbf{y}_{2}$ :

$$
\mathbf{y}=\operatorname{Re} \mathbf{y}+\mathrm{i} \operatorname{Im} \mathbf{y}=\mathbf{y}_{1}+\mathrm{i} \mathbf{y}_{2}
$$

In view of this, the optimization problem $(\mathrm{P})$ is equivalent to

$$
\begin{cases}\min & F\left(\mathbf{y}_{1}, \mathbf{y}_{2}, \sigma\right):=\frac{1}{2}\left\|\mathbf{y}_{1}-\mathbf{y}_{d_{1}}\right\|_{\mathbf{L}^{2}(\Omega)}^{2}+\frac{1}{2}\left\|\mathbf{y}_{2}-\mathbf{y}_{d_{2}}\right\|_{\mathbf{L}^{2}(\Omega)}^{2}+\frac{\varkappa}{2}\|\sigma\|_{L^{2}(\Omega)}^{2} \\ \text { s.t. } & \operatorname{curl} \mu^{-1} \mathbf{c u r l} \mathbf{y}_{1}-\hat{c} \nabla \operatorname{div} \mathbf{y}_{1}-\omega \sigma \mathbf{y}_{2}=\mathbf{j}_{1} \quad \text { in } \Omega \\ & \mathbf{y}_{1} \times \mathbf{n}=0 \quad \text { on } \Gamma \\ & \operatorname{curl} \mu^{-1} \operatorname{curl} \mathbf{y}_{2}-\hat{c} \nabla \operatorname{div} \mathbf{y}_{2}+\omega \sigma \mathbf{y}_{1}=\mathbf{j}_{2} \quad \text { in } \Omega \\ & \mathbf{y}_{2} \times \mathbf{n}=0 \quad \text { on } \Gamma \\ & \operatorname{div} \mathbf{y}_{1}=0 \quad \text { in } \Omega \\ & \operatorname{div} \mathbf{y}_{2}=0 \quad \text { in } \Omega \\ & 0 \leqslant \sigma(x) \leqslant \sigma_{\max } \quad \text { a.e. in } \Omega .\end{cases}
$$

Here, we also use the same notation for the desired state and the applied current density:

$$
\begin{array}{rlrl}
\mathbf{y}_{d_{1}} & :=\operatorname{Re} \mathbf{y}_{d}, \quad \mathbf{y}_{d_{2}}:=\operatorname{Im} \mathbf{y}_{d} \\
\mathbf{j}_{1} & :=\operatorname{Re} \mathbf{j}, & \mathbf{j}_{2} & :=\operatorname{Im} \mathbf{j} .
\end{array}
$$


Analogously, we reformulate the divergence-penalized problem $\left(\mathrm{P}^{\tau}\right)$ as

$$
\begin{cases}\min & F_{\tau}\left(\mathbf{y}_{1}, \mathbf{y}_{2}, \sigma\right):=F\left(\mathbf{y}_{1}, \mathbf{y}_{2}, \sigma\right)+\frac{\tau}{2}\left\|\operatorname{div} \mathbf{y}_{1}\right\|_{L^{2}(\Omega)}^{2}+\frac{\tau}{2}\left\|\operatorname{div} \mathbf{y}_{2}\right\|_{L^{2}(\Omega)}^{2} \\ \text { s.t. } & \operatorname{curl} \mu^{-1} \operatorname{curl} \mathbf{y}_{1}-\hat{c} \nabla \operatorname{div} \mathbf{y}_{1}-\omega \sigma \mathbf{y}_{2}=\mathbf{j}_{1} \quad \text { in } \Omega \\ & \mathbf{y}_{1} \times \mathbf{n}=0 \text { on } \Gamma \\ & \operatorname{curl} \mu^{-1} \operatorname{curl} \mathbf{y}_{2}-\hat{c} \nabla \operatorname{div} \mathbf{y}_{2}+\omega \sigma \mathbf{y}_{1}=\mathbf{j}_{2} \quad \text { in } \Omega \\ & \mathbf{y}_{2} \times \mathbf{n}=0 \text { on } \Gamma \\ & 0 \leqslant \sigma(x) \leqslant \sigma_{\max } \quad \text { a.e. in } \Omega .\end{cases}
$$

Let us remark that SQP-methods are well-known for solving nonlinear optimal control problems. For the details of SQP-methods, we refer to the monographs [15, Section 5.3] and [23, Section 4.11.3]. By a standard linearization of the optimality system for $\left(\mathrm{P}^{\tau}\right)$ (cf. the aforementioned reference), the linear quadratic optimization problem in the $k$ th iteration step of the SQP-algorithm is obtained in the following form:

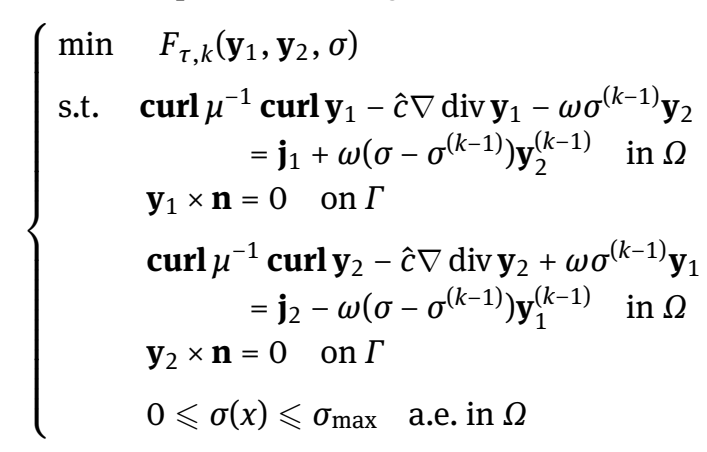

where

$$
\begin{aligned}
F_{\tau, k}\left(\mathbf{y}_{1}, \mathbf{y}_{2}, \sigma\right):= & F_{\tau}^{\prime}\left(\mathbf{y}_{1}^{(k-1)}, \mathbf{y}_{2}^{(k-1)}, \sigma^{(k-1)}\right)\left(\mathbf{y}_{1}-\mathbf{y}_{1}^{(k-1)}, \mathbf{y}_{2}-\mathbf{y}_{2}^{(k-1)}, \sigma-\sigma^{(k-1)}\right) \\
& +\frac{1}{2}\left\|\mathbf{y}_{1}-\mathbf{y}_{1}^{(k-1)}\right\|_{\mathbf{L}^{2}(\Omega)}^{2}+\frac{1}{2}\left\|\mathbf{y}_{2}-\mathbf{y}_{2}^{(k-1)}\right\|_{\mathbf{L}^{2}(\Omega)}^{2}+\frac{\varkappa}{2}\left\|\sigma-\sigma^{(k-1)}\right\|_{L^{2}(\Omega)}^{2} \\
& +\frac{\tau}{2}\left\|\operatorname{div}\left(\mathbf{y}_{1}-\mathbf{y}_{1}^{(k-1)}\right)\right\|_{L^{2}(\Omega)}^{2}+\frac{\tau}{2}\left\|\operatorname{div}\left(\mathbf{y}_{2}-\mathbf{y}_{2}^{(k-1)}\right)\right\|_{L^{2}(\Omega)}^{2} \\
& +\omega\left(\left(\sigma-\sigma^{(k-1)}\right)\left(\mathbf{y}_{1}-\mathbf{y}_{1}^{(k-1)}\right), \mathbf{p}_{2}^{(k-1)}\right)_{\mathbf{L}^{2}(\Omega)} \\
& -\omega\left(\left(\sigma-\sigma^{(k-1)}\right)\left(\mathbf{y}_{2}-\mathbf{y}_{2}^{(k-1)}\right), \mathbf{p}_{1}^{(k-1)}\right)_{\mathbf{L}^{2}(\Omega)}
\end{aligned}
$$

and $\left(\mathbf{y}_{1}^{(k-1)}, \mathbf{y}_{2}^{(k-1)}, \sigma^{(k-1)}\right)$ is an optimal solution of $\left(\mathrm{QP}_{k-1}\right)$ with the associated adjoint state $\left(\mathbf{p}_{1}^{(k-1)}, \mathbf{p}_{2}^{(k-1)}\right)$.

Algorithm 5.1 (SQP-algorithm).

1. Initialization: Choose $\left(\mathbf{y}_{1}^{(0)}, \mathbf{y}_{2}^{(0)}, \sigma^{(0)}, \mathbf{p}_{1}^{(0)}, \mathbf{p}_{2}^{(0)}\right)$ and set $k=1$.

2. Solve the linear quadratic problem $\left(\mathrm{QP}_{k}\right)$ to find the next iterate $\left(\mathbf{y}_{1}^{(k)}, \mathbf{y}_{2}^{(k)}, \sigma^{(k)}, \mathbf{p}_{1}^{(k)}, \mathbf{p}_{2}^{(k)}\right)$.

3. Stop or set $k=k+1$ and go to step 2 .

In our numerical experiment, the computational domain is given by

$$
\Omega=(-0.5,0.5)^{3} \text {. }
$$

Here, (5.1) is triangulated with a regular mesh of mesh size $h$. We employ piecewise constant elements for the control discretization, whereas the state is discretized using continuous (vector-valued) $\mathbb{P}_{1}$-elements. We underline that, since the computational domain (5.1) is convex, the state enjoys $\mathbf{H}^{1}$-regularity (cf. Corollary 3.1). For all computations presented in the following, we use the open source software FEniCS [18] on a PC with a $2.00 \mathrm{Ghz}$ dual core processor and $8 \mathrm{~GB}$ RAM memory. Furthermore, the linear quadratic problem $\left(\mathrm{QP}_{k}\right)$ is solved using a standard projected gradient method (see, e.g., [23, Section 2.12]). The SQP-algorithm is terminated, if $\delta_{k}$ drops below $10^{-4}$, where $\delta_{k}$ is the difference between two iterates of the algorithm given by

$$
\begin{aligned}
\delta_{k}= & \left\|\mathbf{y}_{1}^{(k)}-\mathbf{y}_{1}^{(k-1)}\right\|_{\mathbf{L}^{2}(\Omega)}+\left\|\mathbf{y}_{2}^{(k)}-\mathbf{y}_{2}^{(k-1)}\right\|_{\mathbf{L}^{2}(\Omega)}+\left\|\mathbf{p}_{1}^{(k)}-\mathbf{p}_{1}^{(k-1)}\right\|_{\mathbf{L}^{2}(\Omega)} \\
& +\left\|\mathbf{p}_{2}^{(k)}-\mathbf{p}_{2}^{(k-1)}\right\|_{\mathbf{L}^{2}(\Omega)}+\left\|\sigma^{(k)}-\sigma^{(k-1)}\right\|_{L^{2}(\Omega)} .
\end{aligned}
$$



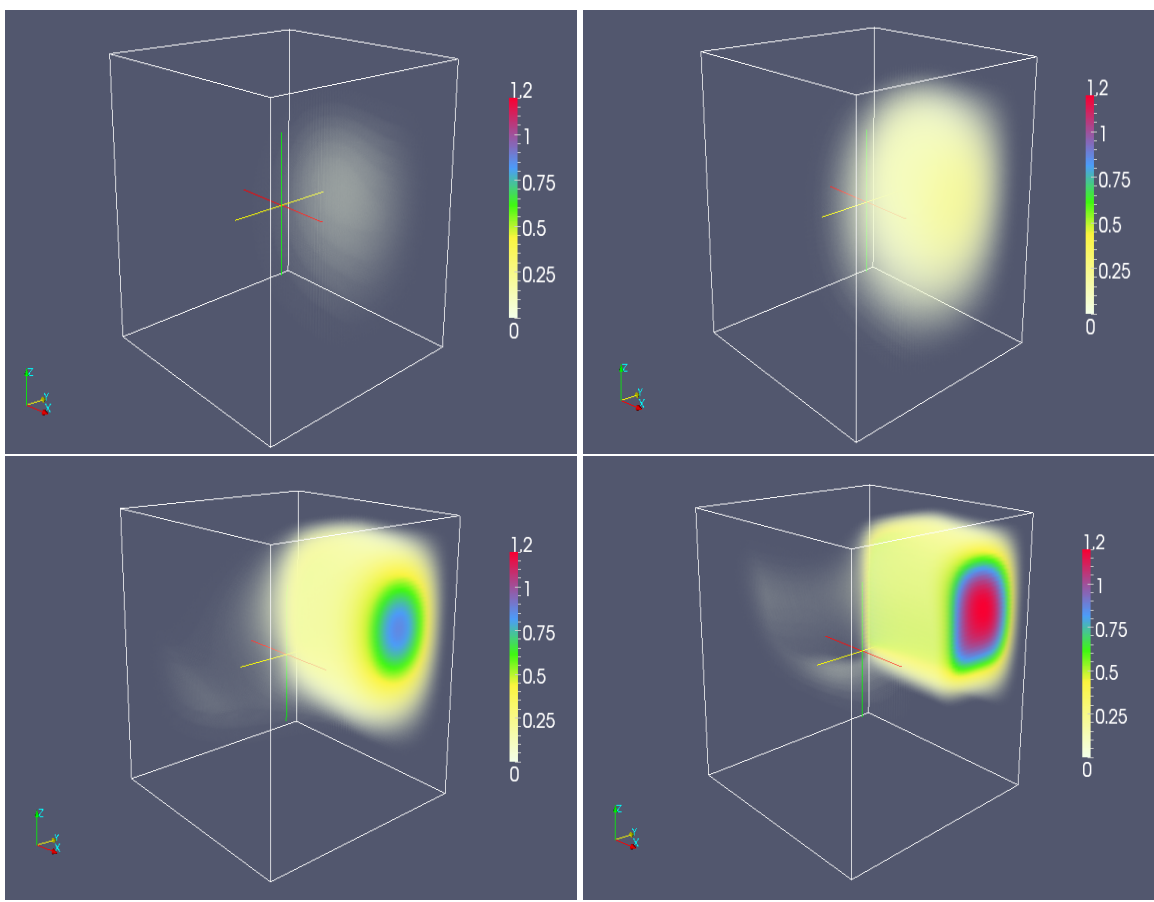

Figure 1. Example 5.1: Computed optimal control with $\tau=10^{2}$ (upper left), $\tau=10^{3}$ (upper right), $\tau=10^{4}$ (lower left), and $\tau=10^{5}$ (lower right).

Example 5.1. We choose $\varkappa=10^{-4}, \mu^{-1}=\hat{c}=10, \omega=1, \sigma_{\max }=100$, and

$$
\begin{aligned}
& \mathbf{y}_{d_{1}}\left(x_{1}, x_{2}, x_{3}\right) \equiv(0,0,0)^{T} \\
& \mathbf{y}_{d_{2}}\left(x_{1}, x_{2}, x_{3}\right)=\left(\left(x_{2}^{2}-0.25\right)\left(x_{3}^{2}-0.25\right), 0,0\right)^{T} .
\end{aligned}
$$

Moreover,

$$
\mathbf{j}_{1}=-\chi_{(0,0.5)^{3}} \mathbf{y}_{d_{2}}, \quad \mathbf{j}_{2}=\operatorname{curl} \mu^{-1} \operatorname{curl} \mathbf{y}_{d_{2}}
$$

where $\chi_{(0,0.5)^{3}}$ denotes the characteristic function on the set $(0,0.5)^{3}$.

Short computations show that the characteristic function $\chi_{(0,0.5)^{3}}$ is the optimal control of $(\mathrm{P})$, if $\varkappa$ vanishes. Therefore, since $\varkappa$ is chosen to be small, the optimal control of $(\mathrm{P})$ is expected to be close to $\chi_{(0,0.5)^{3}}$. We have solved ( $\left.\mathrm{P}^{\tau}\right)$ with a fixed mesh size $h=2^{-6}$ and different values of $\tau$. Figure 1 shows the computed optimal control $\sigma_{h}$ generated by the SQP-algorithm. Here, the region where $\sigma_{h}=0$ is plotted transparently. We observe that, as $\tau$ becomes larger and larger, the computed optimal control $\sigma_{h}$ tends to approximate $\chi_{(0,0.5)^{3}}$.

Example 5.2. We define $K=(-0.5,0)^{3} \cup(0,0.5)^{3}$ and set $\mathbf{j}_{1}=-\chi_{K} \mathbf{y}_{d_{2}}$, where $\mathbf{y}_{d_{2}}$ and all other data are specified as in Example 5.3.

Figure 2 displays the computed optimal control $\sigma_{h}$ with $h=2^{-6}$ generated by the SQP-algorithm. Similarly to the previous example, as $\tau$ increases, the computed optimal control tends to approximate the characteristic function $\chi_{K}$.

\subsection{Numerical test with exact solutions}

We construct exact solutions for the divergence-penalized problem $\left(\mathrm{P}^{\tau}\right)$ by including a shift control $\sigma_{d}$ in the objective functional $F$. More precisely, we redefine $F$ as follows:

$$
F\left(\mathbf{y}_{1}, \mathbf{y}_{2}, \sigma\right):=\frac{1}{2}\left\|\mathbf{y}_{1}-\mathbf{y}_{d_{1}}\right\|_{\mathbf{L}^{2}(\Omega)}^{2}+\frac{1}{2}\left\|\mathbf{y}_{2}-\mathbf{y}_{d_{2}}\right\|_{\mathbf{L}^{2}(\Omega)}^{2}+\frac{\varkappa}{2}\left\|\sigma-\sigma_{d}\right\|_{L^{2}(\Omega)}^{2} .
$$



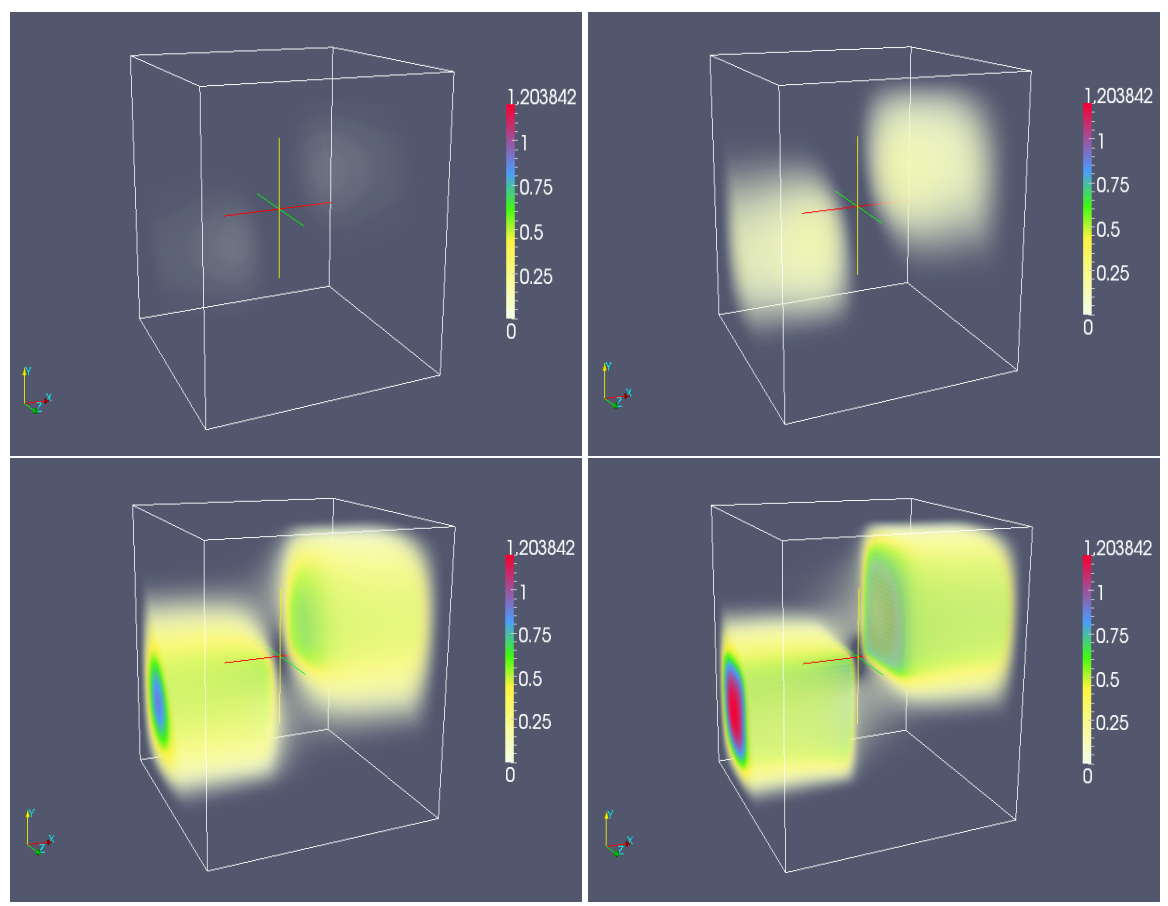

Figure 2. Example 5.2: Computed optimal control with $\tau=10^{2}$ (upper left), $\tau=10^{3}$ (upper right), $\tau=10^{4}$ (lower left), and $\tau=10^{5}$ (lower right).

\begin{tabular}{lcccc}
\hline $\boldsymbol{h}$ & $\mathbf{2}^{-\mathbf{3}}$ & $\mathbf{2}^{-\mathbf{4}}$ & $\mathbf{2}^{-5}$ & $\mathbf{2}^{-6}$ \\
\hline$\# \mathrm{lt}$. & 5 & 5 & 4 & 4 \\
$\left\|\sigma_{h}-\sigma_{d}\right\|_{L^{2}(\Omega)}$ & 2.09002 & 1.54141 & 1.111772 & 0.793758 \\
$\left\|\mathbf{y}_{1, h}-\mathbf{y}_{d_{1}}\right\|_{\mathbf{v}}$ & 0.73200 & 0.37473 & 0.188491 & 0.094388 \\
$\left\|\mathbf{y}_{2, h}-\mathbf{y}_{d_{2}}\right\|_{\mathbf{v}}$ & 0.29903 & 0.15149 & 0.075992 & 0.038026 \\
\hline
\end{tabular}

Table 1. Example 5.3: Convergence of the computed solution.

In the following, we consider two examples, where the triple $\left(\mathbf{y}_{d_{1}}, \mathbf{y}_{d_{2}}, \sigma_{d}\right)$ is constructed to be the optimal solution of $\left(\mathrm{P}^{\tau}\right)$. With the exact solution at hand, we would like to test the convergence performance of the numerical solution computed by the SQP-algorithm.

Example 5.3. We set $\tau=10^{3}, \varkappa=\omega=1, \mu^{-1}=\hat{c}=10, \sigma_{\max }=100$, and

$$
\begin{aligned}
& \mathbf{y}_{d_{1}}\left(x_{1}, x_{2}, x_{3}\right)=\left(\cos \left(\pi x_{2}\right) \cos \left(\pi x_{3}\right), \cos \left(\pi x_{1}\right) \cos \left(\pi x_{3}\right), \cos \left(\pi x_{1}\right) \cos \left(\pi x_{2}\right)\right)^{T} \\
& \mathbf{y}_{d_{2}}\left(x_{1}, x_{2}, x_{3}\right)=10\left(\left(x_{2}^{2}-0.25\right)\left(x_{3}^{2}-0.25\right), 0,0\right)^{T} \\
& \sigma_{d}\left(x_{1}, x_{2}, x_{3}\right)=\left\{\begin{array}{lll}
8, & \text { if } x_{2} \in(-0.25,0), & x_{1} \leqslant 0 \\
10, & \text { if } x_{1} \in(0,0.25), & x_{2} \leqslant 0 \\
15, & \text { if } x_{2} \in(0,0.25), & x_{1} \geqslant 0 \\
20, & \text { if } x_{1} \in(-0.25,0), & x_{2} \geqslant 0 \\
0, & \text { otherwise. }
\end{array}\right.
\end{aligned}
$$

Furthermore, we set

$$
\mathbf{j}_{1}=\operatorname{curl} \mu^{-1} \operatorname{curl} \mathbf{y}_{d_{1}}-\sigma_{d} \mathbf{y}_{d_{2}}, \quad \mathbf{j}_{2}=\operatorname{curl} \mu^{-1} \operatorname{curl} \mathbf{y}_{d_{2}}+\sigma_{d} \mathbf{y}_{d_{1}} .
$$

It is straightforward to check that the optimization problem $\left(\mathrm{P}^{\tau}\right)$ with data specified as in Example 5.3 admits a unique solution $\left(\mathbf{y}_{1}^{\star}, \mathbf{y}_{2}^{\star}, \sigma^{\star}\right)=\left(\mathbf{y}_{d_{1}}, \mathbf{y}_{d_{2}}, \sigma_{d}\right)$. In Table 1, we report on the convergence history of the computed optimal solution. Here, we used zero as initial data, and \#It. denotes the number of iterations required by the SQP-algorithm to converge. We observe that the numerical error in the control (with respect to the $L^{2}(\Omega)$ norm) and the numerical error in the state (with respect to the $\mathbf{V}$-norm) become smaller and smaller as the 


\begin{tabular}{lcccc}
\hline $\boldsymbol{h}$ & $\mathbf{2}^{\mathbf{3}}$ & $\mathbf{2}^{-4}$ & $\mathbf{2}^{-\mathbf{5}}$ & $\mathbf{2}^{-\mathbf{6}}$ \\
\hline EOC $_{\sigma}$ & - & 0.43926 & 0.471389 & 0.486089 \\
EOC $_{\mathbf{y}_{1}}$ & - & 0.96600 & 0.991349 & 0.997823 \\
EOC $_{\mathbf{y}_{2}}$ & - & 0.98105 & 0.995313 & 0.998849 \\
\hline
\end{tabular}

Table 2. Example 5.3: Experimental order of convergence.

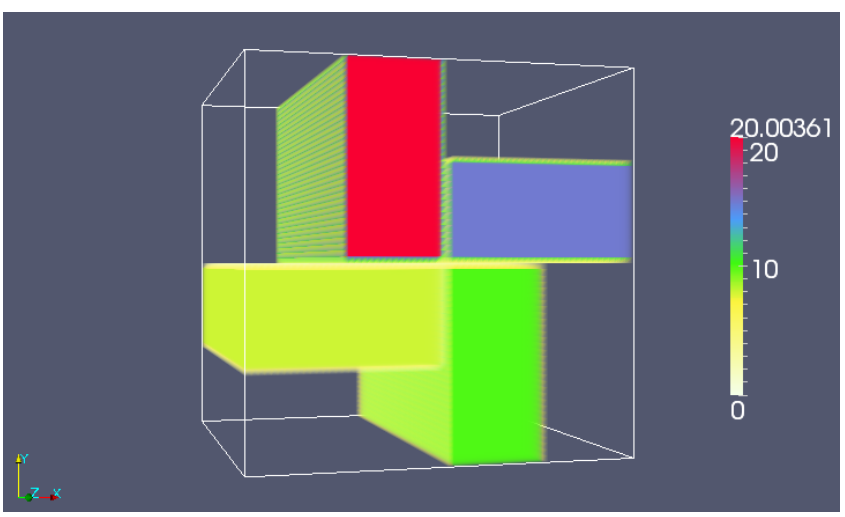

Figure 3. Example 5.3: Computed optimal control.

mesh size $h$ decreases. This is an indication for the convergence of the computed optimal solution towards the exact one. Moreover, to detect the corresponding experimental order of convergence, we use the quantity

$$
\mathrm{EOC}_{\sigma}=\frac{\log \left\|\sigma_{h_{1}}-\sigma_{d}\right\|_{L^{2}(\Omega)}-\log \left\|\sigma_{h_{2}}-\sigma_{d}\right\|_{L^{2}(\Omega)}}{\log h_{1}-\log h_{2}}
$$

for two consecutive mesh sizes $h_{1}$ and $h_{2}$. Similarly, we define $\operatorname{EOC}_{\mathbf{y}_{1}}$ and $\operatorname{EOC}_{\mathbf{y}_{2}}$ for the convergence rate indicators with respect to $\mathbf{y}_{1}$ and $\mathbf{y}_{2}$. The results for these quantities are given in Table 2. Here, we monitor that $\mathrm{EOC}_{\sigma} \approx 0.5$, whereas $\mathrm{EOC}_{\mathbf{y}_{1}}$ and $\mathrm{EOC}_{\mathbf{y}_{2}}$ are very close to 1.0.

Example 5.4. We set $\tau=10^{3}, \varkappa=\omega=1, \mu^{-1}=\hat{c}=2, \sigma_{\max }=100$, and

$$
\begin{aligned}
& \mathbf{y}_{d_{1}}\left(x_{1}, x_{2}, x_{3}\right)=10\left(\left(x_{2}^{2}-0.25\right)\left(x_{3}^{2}-0.25\right), 0,\left(x_{1}^{2}-0.25\right)\left(x_{2}^{2}-0.25\right)\right)^{T} \\
& \mathbf{y}_{d_{2}}\left(x_{1}, x_{2}, x_{3}\right)=10\left(0,\left(x_{1}^{2}-0.25\right)\left(x_{3}^{2}-0.25\right), 0\right)^{T} \\
& \sigma_{d}\left(x_{1}, x_{2}, x_{3}\right)= \begin{cases}10 & \text { in } \Omega_{c}:=\left\{x \in \mathbb{R}^{3} \mid x_{1}^{2}+x_{2}^{2}+x_{3}^{3} \leqslant 1 / 16\right\} \\
0 & \text { otherwise }\end{cases}
\end{aligned}
$$

Moreover, we set

$$
\mathbf{j}_{1}=\operatorname{curl} \mu^{-1} \operatorname{curl} \mathbf{y}_{d_{1}}-\sigma_{d} \mathbf{y}_{d_{2}}, \quad \mathbf{j}_{2}=\operatorname{curl} \mu^{-1} \operatorname{curl} \mathbf{y}_{d_{2}}+\sigma_{d} \mathbf{y}_{d_{1}} .
$$

As in the previous example, the optimization problem $\left(\mathrm{P}^{\tau}\right)$ admits a unique optimal solution $\left(\mathbf{y}_{1}^{\star}, \mathbf{y}_{2}^{\star}, \sigma^{\star}\right)=$ $\left(\mathbf{y}_{d_{1}}, \mathbf{y}_{d_{2}}, \sigma_{d}\right)$. Figure 4 shows the computed optimal control $\sigma_{h}$ with $h=2^{-6}$. Furthermore, the convergence history including the experimental order of convergence are summarized in Table 3. Here, we monitor again that $\mathrm{EOC}_{\sigma} \approx 0.5$ and $\mathrm{EOC}_{\mathbf{y}_{1}}, \mathrm{EOC}_{\mathbf{y}_{2}} \approx 1$. Based on our observation from Example 5.3 and Example 5.4, we experimentally conclude the convergence of orders 0.5 and 1 for the finite element discretization error in the control and the state, respectively. Our goal in the future is to prove this numerical hypothesis and to investigate the finite element analysis in the case of nonconvex polyhedral domains.

\subsection{Numerical test with discontinuous magnetic permeability}

The previous numerical examples consider only a constant magnetic permeability $\mu$. In many real applications, one typically deals with a non-constant magnetic permeability that is possibly highly discontinuous. 


\begin{tabular}{lcccc}
\hline $\boldsymbol{h}$ & $\mathbf{2}^{-\mathbf{3}}$ & $\mathbf{2}^{-\mathbf{4}}$ & $\mathbf{2}^{-5}$ & $\mathbf{2}^{-6}$ \\
\hline$\# \mathrm{It}$. & 5 & 5 & 5 & 5 \\
$\left\|\sigma_{h}-\sigma_{d}\right\|_{L^{2}(\Omega)}$ & 0.61772 & 0.46329 & 0.336368 & 0.239577 \\
$\left\|\mathbf{y}_{1, h}-\mathbf{y}_{d_{1}}\right\|_{\mathbf{v}}$ & 0.42211 & 0.21413 & 0.107453 & 0.053775 \\
$\left\|\mathbf{y}_{2, h}-\mathbf{y}_{d_{2}}\right\|_{\mathbf{v}}$ & 0.29897 & 0.15148 & 0.075990 & 0.038026 \\
EOC $_{\sigma}$ & - & 0.41503 & 0.461898 & 0.489548 \\
EOC $_{\mathbf{y}_{1}}$ & - & 0.97916 & 0.994759 & 0.998692 \\
EOC $_{\mathbf{y}_{2}}$ & - & 0.98093 & 0.995212 & 0.998812 \\
\hline
\end{tabular}

Table 3. Example 5.4: Convergence of the computed solution.

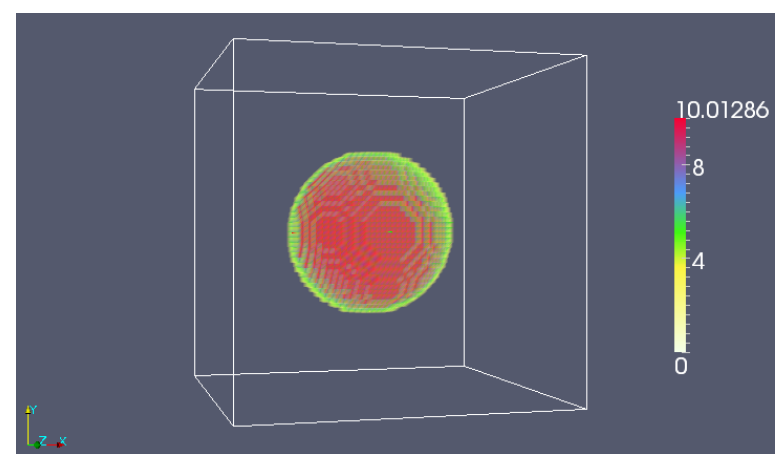

Figure 4. Example 5.4: Computed optimal control.

For instance, if a ferromagnetic metal is considered in an air region, then the magnetic permeability $\mu$ features strong jump discontinuities across the material interface. To test our numerical approach for such a case, we consider the following example.

Example 5.5. We set $\tau=10^{3}, \varkappa=\omega=1, \hat{c}=5, \sigma_{\max }=1000$, and

$$
\sigma_{d}=\left\{\begin{array}{ll}
10 & \text { in } \Omega_{1} \\
10^{2} & \text { in } \Omega_{2} \\
0 & \text { otherwise, }
\end{array} \quad \mu^{-1}= \begin{cases}10^{-3} & \text { in } \Omega_{1} \\
10^{-5} & \text { in } \Omega_{2} \\
1 & \text { otherwise }\end{cases}\right.
$$

where $\Omega_{1}:=\left\{x \in \mathbb{R}^{3} \mid\left(x_{1}-1 / 8\right)^{2}+x_{2}^{2}+x_{3}^{3} \leqslant 1 / 256\right\}$ and $\Omega_{2}:=\left\{x \in \mathbb{R}^{3} \mid\left(x_{1}+1 / 8\right)^{2}+x_{2}^{2}+x_{3}^{3} \leqslant 1 / 256\right\}$. Moreover,

$$
\mathbf{j}_{1}\left(x_{1}, x_{2}, x_{3}\right)=10\left(-x_{2}, x_{1}, 0\right)^{T}, \quad \mathbf{j}_{2} \equiv 0
$$

and $\mathbf{y}_{d_{1}}, \mathbf{y}_{d_{2}}$ are set to be the solution of the state equation with the above data.

Note that $\mu$ is now chosen to have strong jump discontinuities across the interfaces $\partial \Omega_{1}$ and $\partial \Omega_{2}$. As in the previous numerical examples, for $h=2^{-3}, \ldots, 2^{-6}$, the algorithm applied to Example 5.5 successfully converged towards reasonable numerical solutions. This justifies the capability of the proposed optimal control approach for material parameters with jump discontinuities. The computed optimal control at $h=2^{-6}$ is depicted in Fig. 1, which is close to $\sigma_{d}$.

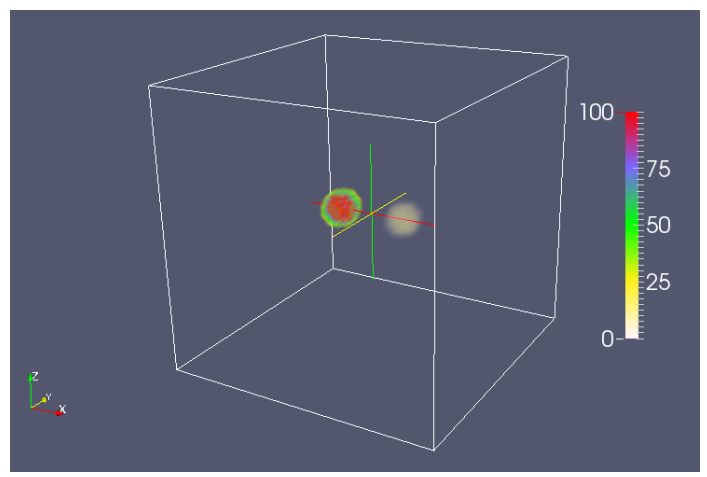

Figure 5. Example 5.5: Computed optimal control. 


\section{References}

[1] A. Alonso and A. Valli, Eddy Current Approximation of Maxwell Equations: Theory, Algorithms and Applications, Springer, 2010.

[2] C. Amrouche, C. Bernardi, M. Dauge, and V. Girault, Vector potentials in three-dimensional non-smooth domains. Mathematical Methods in the Applied Sciences 2 (1998), 823-864.

[3] F. Bachinger, U. Langer, and J. Schöberl, Numerical analysis of nonlinear multiharmonic eddy current problems. Numer. Math. 100 (2005), 593-616.

[4] F. Bachinger, U. Langer, and J. Schöberl, Efficient solvers for nonlinear time-periodic eddy current problems. Comput. Vis. Sci. 9 (2006), 197-207.

[5] R. Beck, R. Hiptmair, R. H. W. Hoppe, and B. Wohlmuth, Residual based a posteriori error estimators for eddy current computation. M2AN Math. Model. Numer. Anal. 34 (2000), 159-182.

[6] C. Caloz and T. Itoh, Electromagnetic Metamaterials: Transmission Line Theory and Microwave Applications.s Wiley-IEEE Press, 2006.

[7] M. Costabel, A remark on the regularity of solutions of Maxwell's equations on Lipschitz domains. Mathematical Methods in the Applied Sciences 12 (1990), 365-368.

[8] M. Costabel and M. Dauge, Weighted regularization of Maxwell equations in polyhedral domains. A rehabilitation of nodal finite elements.s Numer. Math. 93 (2002), 239-277.

[9] M. Costabel, M. Dauge, and S. Nicaise, Singularities of eddy current problems. M2AN Math. Model. Numer. Anal. 37 (2003), 807-831.

[10] P-E. Druet, O. Klein, J. Sprekels, F. Tröltzsch, and I. Yousept, Optimal control of three-dimensional state-constrained induction heating problems with nonlocal radiation effects. SIAM J. Control Optim. 49 (2011), 1707-1736.

[11] H. Feng, D. Jiang, and J. Zou, Simultaneous identification of electric permittivity and magnetic permeability.s Inverse Problems 26 (2010), 095009.

[12] R. Hiptmair, Finite elements in computational electromagnetism. Acta Numerica 11 (2002), 237-339.

[13] R. H.W. Hoppe, Adaptive multigrid and domain decomposition methods in the computation of electromagnetic fields. J. Comput. Appl. Math. (2004) 168, 245-254.

[14] R. H. W. Hoppe, S. I. Petrova, and V. Schulz, Primal-dual Newton-type interior-point method for topology optimization. J. Optim. Theory Appl. 114 (2002), 545-571.

[15] K. Ito and K. Kunisch, Lagrange Multiplier Approach to Variational Problems and Applications.s Society for Industrial and Applied Mathematics, Philadelphia, PA, USA, 2008.

[16] M. Kolmbauer and U. Langer, A robust preconditioned minres solver for distributed time-periodic eddy current optimal control problems.s SIAM J. Sci. Comp. 34 (2012), B785-B809.

[17] R. Leis, Initial-boundary value problems in mathematical physics. B. G. Teubner, Stuttgart, 1986.

[18] A. Logg, K.-A. Mardal, G. N. Wells, et al., Automated Solution of Differential Equations by the Finite Element Method, Springer, 2012.

[19] P. Monk, Finite Element Methods for Maxwell's Equations. Clarendon press, Oxford, 2003.

[20] J. C. Nédélec, Mixed finite elements in $\mathbb{R}^{3}$. Numer. Math. 35 (1980), 315-341.

[21] P. Neittaanmäki, M. Rudnicki, and A. Savini, Inverse Problems and Optimal Design in Electricity and Magnetism.s Oxford University Press, Oxford, 1996.

[22] R. Picard, On the boundary value problems of electro- and magnetostatics. Proc. Royal Soc. Edinburgh, Section: A Mathematics 92 (1982), 165-174.

[23] F. Tröltzsch, Optimal control of partial differential equations. Graduate Studies in Mathematics, American Mathematical Society, Providence, RI, 2010.

[24] F. Tröltzsch and I. Yousept, PDE-constrained optimization of time-dependent 3D electromagnetic induction heating by alternating voltages. ESAIM: M2AN 46 (2012), 709-729.

[25] N. Weck, Maxwell's boundary value problem on Riemannian manifolds with nonsmooth boundaries. J. Math. Anal. Appl. 46 (1974), 410-437.

[26] I. Yousept, Optimal control of quasilinear $\mathbf{H}($ curl)-elliptic partial differential equations in magnetostatic field problems. SIAM J. Control Optim. 51 (2013), No. 5, 3624-3651.

[27] I. Yousept, Optimal control of Maxwell's equations with regularized state constraints. Comput. Optim. Appl. 52 (2012), No. 2, 559-581.

[28] I. Yousept, Finite element analysis of an optimal control problem in the coefficients of time-harmonic eddy current equations, J. Optim. Theory Appl. 154 (2012), No. 3, 879-903.

[29] I. Yousept, Optimal control of a nonlinear coupled electromagnetic induction heating system with pointwise state constraints. Ann. Acad. Rom. Sci. Ser. Math. Appl. 2 (2010), No. 1, 45-77.

[30] J.Zowe and S. Kurcyusz, Regularity and stability for the mathematical programming problem in Banach spaces. Appl. Math. Optim. 5 (1979), 49-62. 